\title{
A Dense-Time Temporal Logic with nice Compositionality Properties
}

\author{
Antonio $\mathrm{Cau}^{1}$ and Willem-Paul de Roever ${ }^{2}$ \\ 1 Software Technology Research Laboratory, \\ Department of Computer Science, \\ De Montfort University, \\ The Gateway, Leicester LE1 9BH, UK \\ cau@dmu.ac.uk \\ 2 Institut für Informatik und Praktische Mathematik, \\ Christian-Albrechts-Universität zu Kiel, \\ Preußerstr. 1-9, D-24105 Kiel, Germany, \\ wpr@informatik.uni-kiel.de
}

\begin{abstract}
A dense temporal logic specification method for the development of reactive systems is introduced. The two development constructs of this method are refinement and composition. A reactive system is specified by a pair consisting of a machine and a condition on the computations of this machine. In order to compose such systems compositionally, each machine step contains additional information such as "this is a system step", or "this is an environment step" or "this is a communication step". Compositionality enables us to break refinement between complex systems into refinement between small and simple systems. The latter can then be verified by existing proof rules for refinement which are reformulated in our formalism.
\end{abstract}

\section{Introduction}

We present a compositional refinement method for reactive systems. A system is called reactive if it maintains some ongoing interaction with its environment, for example an operating system. This contrasts with transformational systems where from some input, without further interaction, output is produced. Because of this characteristic reactive systems are described as sets of behaviours (histories). Here we present a framework which can model both CSP based and shared variable based concurrency, using the work of [Sta84, BKP84, BKP86, DK90, KMP93].

In section 2 reactive systems are specified by sets of histories together with a basis. The latter provides syntactic information about the channels and variables of the specified system. A history is pair consisting of an event and a state function. The domains of these functions are the non-negative real numbers (the underlying dense model). The event function maps each non-negative real number to an event (an action occurring during the operation of the system and 
its environment) and the state function maps each real number to a state of the system and its environment. The intuition is that an occurrence of an action causes (potentially) a state change as illustrated in Figure 1.

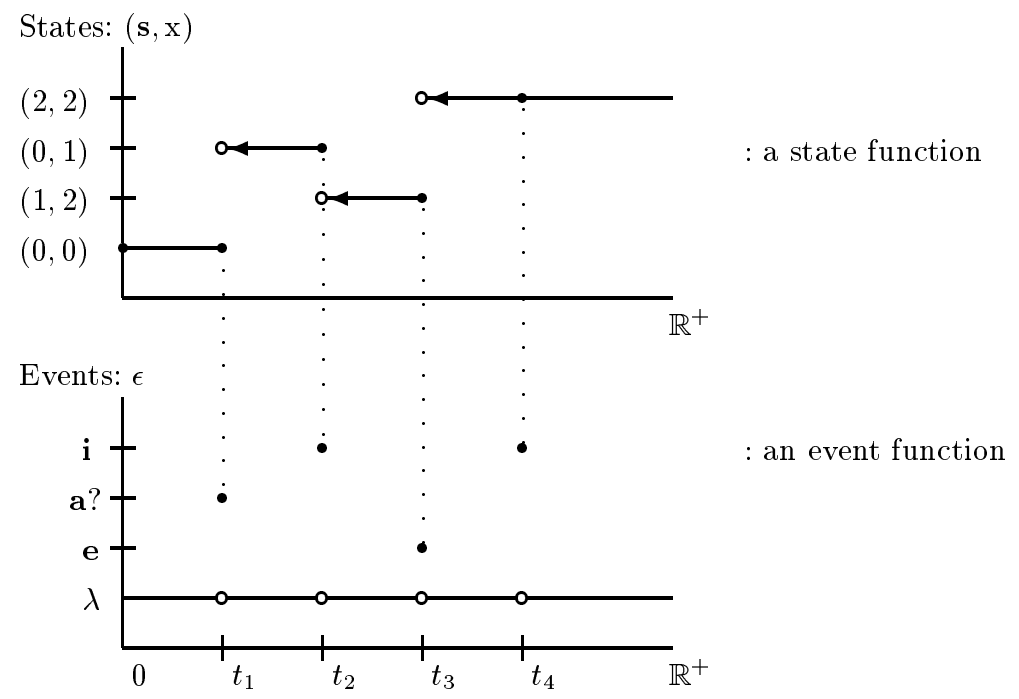

Fig. 1. Illustration of the notion of state and event function, which together characterise the notion of computation of a machine. It illustrates the following computation: initially $(\mathbf{s}, \mathbf{x})=(0,0)$, the event $\mathbf{a}$ ? changes $\mathrm{x}$ into 1 , i.e., $\mathbf{s}$ doesn't change. In the interval $\left[0, t_{1}\right)$ there are only $\lambda$ events. Event $\mathbf{i}$ at point $t_{2}$ changes $(\mathbf{s}, \mathbf{x})$ into $(1,2)$. At point $t_{3}$, the event $\mathbf{e}$ changes $\mathbf{s}$ into 2 and at point $t_{4}$, the event $\mathbf{i}$ doesn't change $\mathbf{s}$ or $\mathrm{x}$.

The use of real numbers as domain for the event and state function handles the stutter problem in refinement. This problem, first observed by Lamport [Lam83], is as follows. Given two behaviours of a system, let the first behaviour contain only consecutive snap-shots of the system that differ from each other whereas the second behaviour, besides containing these same snapshots, contains additional consecutive duplicates of these. This is called stuttering. From the viewpoint of an observer these behaviours are considered as equivalent. Consequently, any formalism that allows to distinguish between these behaviours is not abstract enough and has a power of discrimination which is too strong w.r.t. the criterium of observable behaviour chosen. An example of such a too discriminating formalism is linear temporal logic with the next operator $\bigcirc$. In the present formalism this excessive expressive power is avoided as follows: state changes caused by events happen only now and then, so that in between each two consecutive changes there are uncountably many instants of time at which nothing happens. Consequently, it is impossible to count, or express, stutter steps because the model is "saturated" with them. Furthermore the use of real 
numbers for defining the event and state function enables us to express hiding of variables as existential quantification and consider refinement as implication, even if there are more "states" on the abstract level than on the concrete level. Let the history illustrated in Figure 1 be a history at the abstract level where $\mathrm{x}$ is the variable that should be hidden and let the history illustrated in Figure 2 describe the concrete level. The history of Figure 2 is a refinement of the history of Figure 1.

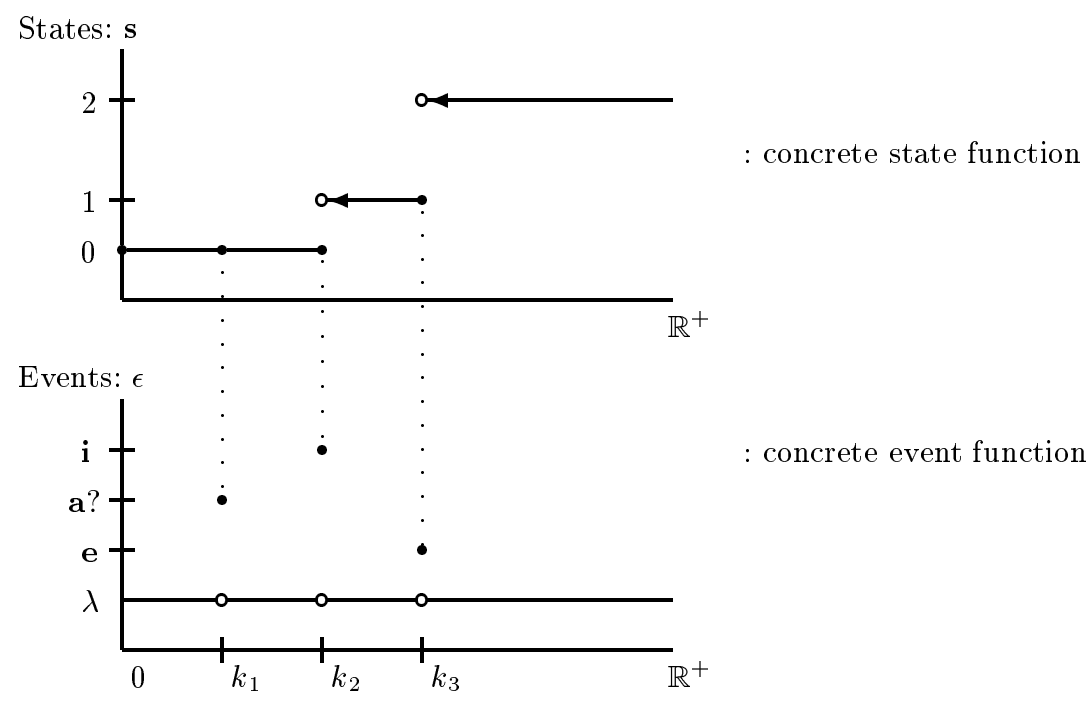

Fig. 2. Illustration of the following concrete computation: initially $\mathbf{s}=(0)$, the event a? doesn't change $\mathbf{s}$, the event $\mathbf{i}$ changes $\mathbf{s}$ into 1 , and event $\mathbf{e}$ changes $\mathbf{s}$ into 2 .

The assumption/commitment approach is used to achieve compositionality in CSP based concurrency [MC81] and shared variable concurrency [Jon83]. These two approaches are unified in [CC96]. These unification ideas are used here, i.e., we use an event variable to store "compositionality information" like "this is a system step" or "this is an environment step" or "this is a communication step". The use of event variable is inspired by the work of [BKP84]. This enables us to describe parallel composition of reactive systems by conjunction. Note that in for instance Lamport's work on TLA [Lam94] this is not always the case: $x:=1 \| x:=1$ must be modelled as disjunction because conjunction leads to a "one process" specification $x:=1$. In our model however, it can be modelled as conjunction because the specification of one component also contains environmental information, especially about the other component. With a "conjoining" operator the histories of both components are merged into a history of the composite one. This conjoining operator, which is based on [CC96], corresponds in our model almost to conjunction. This operator is an extended version of Aczel's parallel composition operator [Acz83], it can also handle CSP based concurrency 
whereas Aczel's can only handle shared variable based concurrency.

We also investigate how composition relates to refinement, i.e., in fact we obtain the notion of compositional refinement [ZCdR92]. Compositional refinement means intuitively that if the components of an abstract composed system are refined by the components of a concrete composed system then the total abstract composed system is refined by the total concrete composed system, i.e., refinement is preserved under composition. This enables the reduction of refinement problems for large, complex, systems to a number of refinement problems between small, simple, systems. In fact without this property we wouldn't have been able to prove correctness of some complicated examples like stable storage and Dijkstra's solution to the readers and writers problem.

The dense temporal logic DTL based on histories is introduced in section 2.2. This logic is based on [Sta84, BKP86, DK90, KMP93]. A salient feature of this logic is the "immediately after" operator "'", in a version which is stutter insensitive. The logic is used to (1) specify our systems, (2) express refinement and composition between systems, and (3) verify refinement between systems.

We will apply our formalism to an simple example. In [Cau95] the formalism has been applied to prove correctness of a fault tolerant system implementing stable storage and Dijkstra's solution of the readers and writers problem. The full proof of those examples and all the proofs of the lemmas and the theorems given in this paper can be found in [Cau95].

\section{Specification of Reactive Systems}

This section explains how reactive systems can be specified. Firstly they will be specified at the semantical level, i.e., by sets of histories. A history intuitively specifies which event occurs at a particular point and in what state the system is at that particular point. Secondly reactive systems are specified using the dense temporal logic DTL.

\subsection{Semantic Specification of Reactive Systems}

Reactive systems will be specified by sets of histories. A history is a pair consisting of an event function and a state function. An event function records at each point (i.e., for each positive real, including zero) which event occurs. An event is an instantaneous occurrence of an action during the operation of a system, that can be generated by that system or by its environment and that is of interest at the given level of abstraction. Four kinds of actions are distinguished:

1. Communication actions a?, b!, i.e., actions that transmit information over a channel. A channel is a connection between the system and its environment.

2. System actions i, i.e., non-communication actions of the system.

3. Environment actions e, i.e., non-communication actions of the environment.

4. Silent actions $\lambda$, i.e., actions that don't influence the status of the system. 
Event states are introduced in order to record which event occurs during the operation of the system. An event state corresponds to the usual notion of state in that instead of normal program variables event variables are used.

Definition 1 (Event state) Let Chan denote a nonempty set of channels names. Let $\mathfrak{E}$ denote the set of event variables with typical elements $\epsilon, \epsilon_{0}, \epsilon_{1}, \ldots$ Event variable $\epsilon$ will record which action occurs during the operation of the system, and the event variables $\epsilon_{0}, \epsilon_{1}, \ldots$ are auxiliary event variables recording which actions occur in components of the system. Let $\mathfrak{A}$ denote the set of actions, with typical elements $\mathbf{i}$ (denoting system actions), e (denoting environment actions), $\mathbf{a} ?, \mathbf{b} !^{3}$... denoting respectively an input communication action over channel $\mathbf{a}$ and an output communication action over channel $\mathbf{b}$, and $\lambda$ denoting the silent action. An event state is a mapping $\delta$ from $\mathfrak{E}$ to $\mathfrak{A}$. Let $\Delta$ denote the set of all event states.

A state function records at each point (a non-negative real number) the process state, i.e., the usual notion of state of a system and its environment. In order to distinguish the normal variables from the event variables the normal variables are called here process variables. Three kind of process variables are distinguished:

1. Shared process variables which are "shared" between a system and its environment.

2. Local process variables which are only accessible by a system.

3. Rigid variables which are not changed by the system and its environment, i.e., which are only used for specification purposes.

Definition 2 (Process state) A process state is a mapping from variables to values. Let $\mathfrak{V}$ denote the set of shared variables with typical elements $\mathbf{s}, \ldots$, and $\mathfrak{X}$ the set of local variables $(\mathfrak{V} \cap \mathfrak{X}=\emptyset$ ) with typical elements $\mathrm{x}, \ldots$, and $\mathfrak{R}$ the set of rigid variables with typical elements $n, \ldots$ A state is a mapping $\sigma$ from $\mathfrak{V} \cup \mathfrak{X} \cup \mathfrak{R}$ to the set of values Val. Let $\Sigma$ denote the set of all process states.

As already said above, event and state functions are mappings from the nonnegative reals to, respectively event and process states. Because of this strategy, some requirements are needed in order to specify "reasonable" histories. Here "reasonable" is used in the sense that in a bounded interval only a finite number of non-silent actions and process state changes can occur. This requirement is called the finite variability condition [BKP86]. Next several notions for functions from $\mathbb{R}^{+}$(the positive reals including 0 ) to some domain $D$ are introduced in order to define this requirement and to formally define the notions of event and state functions.

Definition 3 ((Dis)continuous) Given function $f: \mathbb{R}^{+} \rightarrow D . f$ is called left continuous, if $\forall t>0 . f(t)=\lim _{t_{1} \rightarrow t} f\left(t_{1}\right)$, right continuous, if $\forall t \geq 0$. $f(t)=\lim _{t \leftarrow t_{1}} f\left(t_{1}\right)$,

\footnotetext{
${ }^{3}$ In this paper we omit the value part of the communication, i.e., which value is transmitted, in order to ease the formalism a little bit.
} 
discontinuous at $t$, if $f(t) \neq \lim _{t_{1} \rightarrow t} f\left(t_{1}\right) \vee f(t) \neq \lim _{t \leftarrow t_{1}} f\left(t_{1}\right)$, strongly discontinuous at $t$, if $f(t) \neq \lim _{t_{1} \rightarrow t} f\left(t_{1}\right) \wedge f(t) \neq \lim _{t \leftarrow t_{1}} f\left(t_{1}\right)$. $f$ has the finite variability property iff $f$ has only finitely many points of discontinuity in any interval $[a, b], 0 \leq a \leq b, a, b \in \mathbb{R}^{+}$.

Now event and state functions can be defined. In [DK90] it is explained why initial stuttering is needed to express refinement in a logic with the help of existential quantification and implication. To adopt this result, we must first define what notion of stuttering in our setting corresponds with the one of [DK90]. Within our setting a stutter step is regarded as a step in which a non-communication action doesn't change the state. As such initial stuttering can expressed by requiring that in the first interval the event function has the constant value $\lambda$ and the state function remains constant. Furthermore a state should remain constant for some continuous interval of points in order to be observable. Also non- $\lambda$ events are considered to be single points. Another possibility would be for the events to remain constant during an interval of points. The intuitive meaning of a history is that the points at which non- $\lambda$ events occur mark the state changes. For these non- $\lambda$ events the question to be answered is: At which point of the interval should the state change take place? Answer: at the last point of the interval which describes the event. So for events only the last point of the interval is interesting because it marks the state change. So why consider an interval if only its last point is interesting? This is explained by our choice for letting non- $\lambda$ events occur only at single points, as captured by the following definitions.

\section{Definition 4 (restriction)}

For $g: A_{1} \rightarrow A_{2}, A_{0} \subseteq A_{1}$ define $\left.g\right|_{A_{0}} ^{1}: A_{0} \rightarrow A_{2}$ as $\left.g\right|_{A_{0}} ^{1}(x)=g(x)$ for $x \in A_{0}$. For $g: A_{1} \rightarrow\left(A_{2} \rightarrow A_{3}\right), A_{0} \subseteq A_{2}$ define $\left.g\right|_{A_{0}} ^{2}: A_{1} \rightarrow\left(A_{0} \rightarrow A_{3}\right)$ as $\left.g\right|_{A_{0}} ^{2}(t)(x)=g(t)(x)$ for $x \in A_{0}$.

Definition 5 (Event function) An event function $\psi$ is a function from $\mathbb{R}^{+}$ to $\Delta$, such that $\left.\psi\right|_{\epsilon} ^{2}$ has the finite variability condition, $\psi(0)(\epsilon)=\lambda$ (i.e., initial stuttering) and for all points $t, \psi$ is strongly discontinuous at $t$ iff $\psi(t)(\epsilon) \neq \lambda$ (i.e., an event function is almost constant $\lambda$ ). Let $\Psi$ denote the set of all event functions.

Figure 1 illustrates the notion of event function. At point $t_{1}$ event $\mathbf{a}$ ? occurs, at point $t_{2}$ event $\mathbf{i}$ occurs, at point $t_{3}$ event $\mathbf{e}$ occurs, and at all other points event $\lambda$ occurs. Points $t_{1}, t_{2}$ and $t_{3}$ are here the strongly discontinuous points.

Definition 6 (State function) $A$ state function $\theta$ is a left continuous function from $\mathbb{R}^{+}$to $\Sigma$ such that for all $n \in \mathfrak{R}$ and $t \in \mathbb{R}^{+}, \theta(t)(n)=\theta(0)(n)$ (i.e., the rigid variables don't change at all), and for all $x \in \mathfrak{V} \cup \mathfrak{X},\left.\theta\right|_{x} ^{2}$ satisfies the finite variability property and $\left.\theta\right|_{x} ^{2}(0)(x)=\left.\lim _{0 \leftarrow t_{1}} \theta\right|_{x} ^{2}\left(t_{1}\right)(x)$ (i.e., initial stuttering). Let $\Theta$ denote the set of all state functions.

Figure 1 illustrates the notion of state function. In interval $\left[0, t_{1}\right]$ the system is in state $(\mathbf{s}, \mathrm{x})=(0,0)$, in interval $\left(t_{1}, t_{2}\right]$ in state $(\mathbf{s}, \mathrm{x})=(0,1)$, in interval $\left(t_{2}, t_{3}\right]$ 
in state $(\mathbf{s}, \mathbf{x})=(1,2)$ and in interval $\left(t_{2}, \infty\right)$ in state $(\mathbf{s}, \mathrm{x})=(2,2)$. The event $\mathbf{i}$ at $t_{4}$ is an illustration of a non- $\lambda$ stutter step.

The following definition combines the notions of state function and event function into the notion of history. Two requirements are imposed on the combination of event and state function for it to result in a history. The first requirement is that silent actions don't give rise to process state changes. The second requirement is that communication actions don't change the shared variables; this requirement is imposed in order to model CSP [Hoa84] like processes.

Definition 7 (History) $A$ history $h$ is a pair $\langle\psi, \theta\rangle$, where $\psi$ is an event function and $\theta$ is a state function s.t. a $\lambda$ action doesn't change the values of variables from $\mathfrak{V} \cup \mathfrak{X}$, i.e., $\forall t: \psi(t)(\epsilon)=\lambda \rightarrow \theta(t)=\lim _{t \leftarrow t_{1}} \theta\left(t_{1}\right)$ and a communication action doesn't change the values of shared variables, i.e., $\forall t: \psi(t)(\epsilon)=$ $\mathbf{a} ? \vee \psi(t)(\epsilon)=\left.\mathbf{a} ! \rightarrow \theta(t)\right|_{\mathfrak{V}} ^{1}=\left.\lim _{t \leftarrow t_{1}} \theta\left(t_{1}\right)\right|_{\mathfrak{V}} ^{1}$. Let $\mathcal{H}$ denote the set of all histories.

The following definition defines when a history is stutter equivalent to another history. A history collapse function $\natural_{\mathfrak{h}}(h)$ is introduced that takes a history and collapses it in such a way that the non-stutter steps only occur at discrete points (elements of $\mathbb{N}$ ) and at all remaining points stutter steps occur. Also a restricted version of the history stutter equivalence relation is defined, namely, restricted to the process state information. The last one will be used to define a "process state history stutter insensitive" logic DTL. Restricted to a special kind of formulae we obtain the "stutter insensitive" logic.

Definition 8 (History collapse, stutter equivalent) Given history $h \in \mathcal{H}$, the history collapse denoted $\bigsqcup_{\mathfrak{h}}(h)$ is a function from $\mathcal{H}$ to $\mathcal{H}$ defined as $\natural_{\mathfrak{h}}(h) \doteq$ $h \circ \operatorname{di}(h)$ where $\operatorname{di}(h)$ is the discretisation bijection for $h$ from $\mathbb{R}^{+}$to $\mathbb{R}^{+}$and is defined as follows:

Let $\operatorname{tt}(h, k)$ be the function from $\mathcal{H} \times \mathbb{N}$ to $\mathbb{R}^{+}$that gives the point in $\mathbb{R}^{+}$of the $k$-th change in $h$, formally:

$\operatorname{tt}(h, 0)=0$
$\operatorname{tt}(h, k+1) \doteq \min \left(t \mid t>\operatorname{tt}(h, k) \wedge\left(\psi(t)(\epsilon) \notin\{\lambda, \mathbf{i}, \mathbf{e}\} \vee \theta(t) \neq \lim _{\operatorname{tt}(h, k) \leftarrow t_{1}} \theta\left(t_{1}\right)\right)\right)$

Let $n n(h)$ denote the number of non-stutter points of $h$. Then the discretisation bijection $\operatorname{di}(h)$ for $h$ is defined as follows:

$$
\begin{aligned}
& \operatorname{di}(h)(t) \doteq \\
& \begin{cases}\operatorname{tt}(h, k)+(t-k) *(\operatorname{tt}(h, k+1)-\operatorname{tt}(h, k)) & n n(h)<\infty \wedge 0 \leq k<n n(h) \\
& \wedge k \leq t \leq k+1 \\
\operatorname{tt}(h, k)+(t-k) & n n(h)<\infty \wedge k=n n(h) \wedge k \leq t \\
\operatorname{tt}(h, k)+(t-k) *(\operatorname{tt}(h, k+1)-\operatorname{tt}(h, k)) & n n(h)=\infty \wedge 0 \leq k \\
& \wedge k \leq t \leq k+1\end{cases}
\end{aligned}
$$

The inverse discretisation of $h$ is denoted $\mathrm{di}^{-1}(h)$. Given histories $h_{0}, h_{1} \in \mathcal{H}$, $h_{0}$ is history stutter equivalent to $h_{1}$ denoted $h_{0} \simeq_{h} h_{1}$ iff $n n\left(h_{0}\right)=n n\left(h_{1}\right)$ 
and $\theta_{\mathfrak{\natural}_{\mathfrak{h}}\left(h_{0}\right)}=\theta_{\mathfrak{\natural}_{\mathfrak{h}}\left(h_{1}\right)}$ and $\psi_{\mathfrak{\natural}_{\mathfrak{h}}\left(h_{0}\right)}(k)=\psi_{\mathfrak{\natural}_{\mathfrak{h}}\left(h_{1}\right)}(k), k \leq n n\left(h_{0}\right)$, i.e., the number of non-stutter steps should be equal, the state information should be equal in both collapsed histories and the event information should be equal in the points of non-stuttering. $h_{0}$ is history process state stutter equivalent to $h_{1}$ denoted $h_{0} \simeq_{\theta_{h}} h_{1}$ iff $n n\left(h_{0}\right)=n n\left(h_{1}\right)$ and $\theta_{\mathfrak{\natural}_{\mathfrak{h}}\left(h_{0}\right)}=\theta_{\mathfrak{七}_{\mathfrak{h}}\left(h_{1}\right)}$, i.e., only the process state information is considered.

These notions are best understood by an example. What's done is that by stretching, resp., compressing an interval as a rubber string, the non-stuttering events are made to occur at an initial interval of the positive natural numbers, as shown in Figure 3.

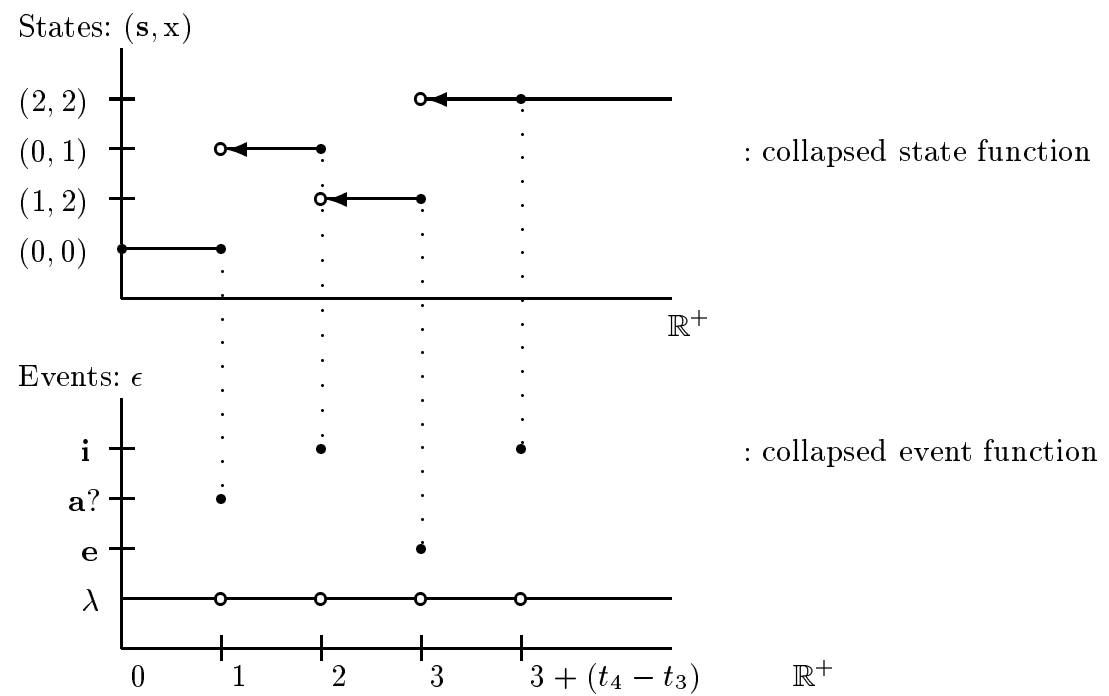

Fig. 3. Illustration of the collapsed history of Figure 1.

The basis provides syntactic info and consists of a process basis, specifying the local and shared variables of the system, and a action basis which specifies the input and output communication channels of a system. The following definition introduces the notions of basis and history sets. The latter constrain a specific process basis, i.e., specific sets of shared variables and local variables are constrained to change in specific ways, whereas the variables outside this process basis can change without restriction, with exception of the rigid variables which do not change at all.

Definition 9 (Basis, history set constraining a basis) $A$ basis (denoted by $B$ ) is a pair $\left(B^{A}, B^{P}\right)$, where $B^{A}$ (called action basis) is a pair (In, Out) where In is a set of input communication channels and Out is a set of output communication channels, and where $B^{P}$ (called process basis) is a tuple $(\mathrm{V}, \mathrm{X})$ where $\mathrm{V}$ a finite set of shared variables and $\mathrm{X}$ a finite set of local variables. Given a 
history $h \in \mathcal{H}$ and process basis $B^{P}$, the process basis restriction of $h$ denoted $\left.h\right|_{B^{P}} ^{2}$ is defined as $\left\langle\psi,\left.\theta\right|_{\mathrm{V} \cup \mathrm{X}} ^{2}\right\rangle$. Given a set of histories $H$ and process basis $B^{P}$, $H$ is constrained by $B^{P}$ iff $\forall h_{1}, h_{2} \in \mathcal{H}:\left.h_{1}\right|_{B^{P}} ^{2}=\left.h_{2}\right|_{B^{P}} ^{2} \rightarrow\left(h_{1} \in H \leftrightarrow h_{2} \in H\right)$.

Next we introduce the notion of history specification as a pair consisting of a basis and a set of histories constraining the process basis.

Definition 10 (History specification of a system) $A$ history specification of a system (denoted $\mathcal{S}$ ) is a pair $(B, H)$ where $B$ is a basis and $H$ is a set of histories constraining process basis $B^{P}$ such that an environment action $\mathbf{e}$ doesn't change the local variables of the system: $\forall t: \psi(t)(\epsilon)=\left.\mathbf{e} \rightarrow \theta(t)\right|_{\mathrm{X}} ^{1}=$ $\left.\lim _{t \leftarrow t_{1}} \theta\left(t_{1}\right)\right|_{\mathrm{X}} ^{1}$.

We also borrow several notions from topology for the definition of safety and liveness sets of histories, based on [AS85]. Informally, a safety set of histories consists of histories where nothing "bad" happens and a liveness set of histories consists of histories where something "good" eventually happens.

Definition 11 (Safety and liveness set) Let $H$ be a set of histories and $h \in$ $\mathcal{H}$.

The prefix of $h$ of length $t$, denoted $\left.h\right|_{t}$, is defined as

$$
h l_{t}\left(t_{0}\right) \doteq\left\{\begin{array}{l}
\left\langle\psi\left(t_{0}\right), \theta\left(t_{0}\right)\right\rangle \quad 0 \leq t_{0} \leq t \\
\langle\psi(0), \theta(t)\rangle \quad t_{0}>t
\end{array}\right.
$$

The distance function $d$ from $\mathcal{H} \times \mathcal{H} \rightarrow \mathbb{R}^{+}$is defined as:

$$
d\left(h_{1}, h_{2}\right) \doteq \begin{cases}0 & \text { if } h_{1}=h_{2} \\ 1 & \text { if } h_{1}(0) \neq h_{2}(0) \\ 2^{-\sup \left\{t \in \mathbb{R}^{+}\left|h_{1}\right|_{t}=\left.h_{2}\right|_{t}\right\}} & \text { otherwise }\end{cases}
$$

$H$ is called $d$-open iff $\forall h \in H: \exists \varepsilon>0: \forall h_{1}: d\left(h, h_{1}\right)<\varepsilon \rightarrow h_{1} \in H$.

The topology with $\{H \subseteq \mathcal{H} \mid H$ is d-open $\}$ as its basis is called the $d$ induced topology of $(\mathcal{H}, d)$ denoted $\tau_{d}$.

$H$ is called a $\tau_{d}$-environment of $h$ iff $\exists H_{1} \in \tau_{d}: h \in H_{1} \wedge H_{1} \subseteq H$.

The interior of $H$ denoted in $(H)$ is defined as $\left\{h \in \mathcal{H} \mid H\right.$ is a $\tau_{d}$ environment of $\left.h\right\}$.

The closure of $H$ denoted $c l(H)$ is defined as $\mathcal{H} \backslash($ in $(\mathcal{H} \backslash H))$.

$H$ is a safety set iff $c l(H)=H$. $H$ is a liveness set iff $c l(H)=\mathcal{H}$.

Note: the only set that is both a safety and a liveness set is $\mathcal{H}$ [AS85].

Following Abadi and Lamport [AL91], a specification method for systems uses the notion of machine. A machine consists of a set of states and a statetransition relation. Our intention is that the set of computations (i.e. histories) of a machine used for describing a system should correspond to the history specification of this system. A machine however can only generate safety sets of histories [AS87]. Therefore, a liveness set is specified as a condition on the set of computations (histories) of a machine. Next the formal definition of a machine is given. 
Definition 12 (Machine) The machine specification $M$ of a system is a triple $(B, I, T)$ where:

- B: the basis of $M$; a tuple ((In, Out), $(\mathrm{V}, \mathrm{X}))$. Note: the shared variables will be printed in bold faced style in order to distinguish them from the local variables.

- I : a non-empty subset of $\Sigma$, the set of initial states, such that

- $\forall \sigma_{0}, \sigma_{1} \in \Sigma:\left(\left.\sigma_{0}\right|_{\mathrm{V} \cup \mathrm{X}} ^{1}=\left.\sigma_{1}\right|_{\mathrm{V} \cup \mathrm{X}} ^{1}\right) \rightarrow\left(\sigma_{0} \in I \leftrightarrow \sigma_{1} \in I\right)$, i.e., it constrains the variables from $\mathrm{V} \cup \mathrm{X}$ only.

- $T$ : the state-transition relation (finite), $T \subseteq \Delta \times \Sigma^{2}$, such that

- $\forall \sigma_{0}, \sigma_{1} \in \Sigma, \delta \in \Delta:\left.\left\langle\delta, \sigma_{0}, \sigma_{1}\right\rangle \in T \rightarrow \sigma_{0}\right|_{\mathfrak{R}} ^{1}=\left.\sigma_{1}\right|_{\mathfrak{R}} ^{1}$, i.e., the rigid variables don't change at all.

- $\forall \sigma_{0}, \sigma_{1}, \sigma_{2}, \sigma_{3} \in \Sigma, \delta \in \Delta:\left(\left.\sigma_{0}\right|_{\mathrm{V} \cup \mathrm{X}} ^{1}=\left.\left.\sigma_{2}\right|_{\mathrm{V} \cup \mathrm{X}} ^{1} \wedge \sigma_{1}\right|_{\mathrm{V} \cup \mathrm{X}} ^{1}=\left.\sigma_{3}\right|_{\mathrm{V} \cup \mathrm{X}} ^{1}\right) \rightarrow$ $\left(\left\langle\delta, \sigma_{0}, \sigma_{1}\right\rangle \in T \leftrightarrow\left\langle\delta, \sigma_{2}, \sigma_{3}\right\rangle \in T\right)$, i.e. $T$ constrains $B^{P}$ only.

- $\forall \sigma_{0}, \sigma_{1} \in \Sigma, \delta \in \Delta:\left.\left(\left\langle\delta, \sigma_{0}, \sigma_{1}\right\rangle \in T \wedge(\delta(\epsilon)=\mathbf{a} ? \vee \delta(\epsilon)=\mathbf{a} !)\right) \rightarrow \sigma_{0}\right|_{\mathrm{V}} ^{1}=$ $\left.\sigma_{1}\right|_{\mathrm{V}} ^{1}$, i.e., a communication action doesn't change the values of shared variables, and

- $\forall \sigma_{0}, \sigma_{1} \in \Sigma, \delta \in \Delta:\left.\left(\left\langle\delta, \sigma_{0}, \sigma_{1}\right\rangle \in T \wedge \delta(\epsilon)=\mathbf{e}\right) \rightarrow \sigma_{0}\right|_{\mathrm{X}} ^{1}=\left.\sigma_{0}\right|_{\mathrm{X}} ^{1}$, i.e., an environment action doesn't change the values of local variables of the system.

- $\forall \sigma_{0}, \sigma_{1} \in \Sigma, \delta \in \Delta:\left\langle\delta, \sigma_{0}, \sigma_{1}\right\rangle \in T \rightarrow\left(\delta(\epsilon) \notin\{\lambda, \mathbf{i}, \mathbf{e}\} \vee \sigma_{0} \neq \sigma_{1}\right)$, i.e., no stutter transitions are specified.

Example 1. $M=(B, I, T)$ where the basis $B \doteq((\{\mathbf{a}\}, \emptyset),(\{\mathbf{v}\},\{\mathrm{u}\}))$, the initial states $I \doteq\{\sigma \in \Sigma \mid \sigma(\mathrm{u})=0$ and $\sigma(\mathbf{v})=0\}$ and the transition relation $T \doteq\left\{\left\langle\delta, \sigma_{0}, \sigma_{1}\right\rangle \in \Delta \times \Sigma^{2} \mid\right.$

1. $\left(\delta(\epsilon)=\mathbf{a}\right.$ ? and $\sigma_{0}(\mathrm{u})=0$ and $\sigma_{1}(\mathrm{u})=1$ and $\left.\sigma_{1}(\mathbf{v})=\sigma_{0}(\mathbf{v})\right)$ or

2. $\left(\delta(\epsilon)=\mathbf{i}\right.$ and $\sigma_{0}(\mathrm{u})=1$ and $\sigma_{0}(\mathbf{v})=1$ and $\sigma_{1}(\mathrm{u})=2$ and $\left.\sigma_{1}(\mathbf{v})=0\right)$ or

3. $\left(\delta(\epsilon)=\mathbf{e}\right.$ and $\sigma_{1}(\mathrm{u})=\sigma_{0}(\mathrm{u})$ and $\left.\left.\sigma_{1}(\mathbf{v})=\sigma_{0}(\mathbf{v})+1\right)\right\}$

The concepts of event and state functions are related by the notion of computation of a machine $M$. A computation of $M$ intuitively expresses that an event function and a state function fit together in that at any point $t$ any triple consisting of (1) the event occurring at $t,(2)$ the state just before and including $t$, and (3) the state just after $t$, belongs to the state transition relation of $M$ (see fig. 1). Because such a relation doesn't contain stutter steps but histories do, a set of stutter transitions should be defined in order to relate machine computations to histories.

Definition 13 (Computation) Let $h=\langle\psi, \theta\rangle \in \mathcal{H}, t \in \mathbb{R}^{+}$and $M=(B, I, T)$. The step occurring at $t$ in $h$ is defined as: $\operatorname{Step}_{h}(t) \doteq\left\langle\psi(t), \theta(t), \lim _{t \leftarrow t_{1}} \theta\left(t_{1}\right)\right\rangle$. The set of stutter steps, is defined as $\mathrm{STU} \doteq\left\{\left\langle\delta, \sigma_{0}, \sigma_{1}\right\rangle \mid \delta(\epsilon) \in\{\lambda, \mathbf{i}, \mathbf{e}\} \wedge \sigma_{0}=\right.$ $\left.\sigma_{1}\right\}$.

$A$ computation of $M$ is a history $h$ s.t. $\theta(0) \in I$ and $\forall t:$ Step $_{h}(t) \in T \vee$ $\operatorname{Step}_{h}(t) \in \mathrm{STU}$.

Comp $(M)$ denotes the set of all computations of $M$. 
Lemma 1 (Machine is safety) Let $M$ be a machine then $\operatorname{Comp}(M)$ is a safety set.

The machine specification of a system now consists of a machine $M$ and a set of histories $L$ constraining the basis of this machine such that the closure of the intersection of $\operatorname{Comp}(M)$ and $L$ equals $\operatorname{Comp}(M)$. This is the machine closedness property of a system specification introduced in [AFK88, AL91]. Let $A \rightarrow B$ denote $\bar{A} \cup B$. By a result of [AS85] every set of histories can be written as the intersection of a safety set and a liveness set namely $\operatorname{cl}(\operatorname{Comp}(M) \cap$ $L) \bigcap \operatorname{cl}(\operatorname{Comp}(M) \cap L) \rightarrow(\operatorname{Comp}(M) \cap L)$. By the machine closedness property this can be written as $\operatorname{Comp}(M) \bigcap \operatorname{Comp}(M) \rightarrow L$. This implies that $\operatorname{Comp}(M)$ specifies the safety properties and $\operatorname{Comp}(M) \rightarrow L$ the liveness properties of the system.

Definition 14 (Machine specification) $A$ machine specification $\mathcal{S}$ of a system is a pair $(B, \operatorname{Comp}(M) \cap L)$ where $M$ is a machine with basis $B$ and $L$ a set of histories constraining only $B^{P}$ such that $\operatorname{cl}(\operatorname{Comp}(M) \cap L)=\operatorname{Comp}(M)$. The set of computations of $\mathcal{S}$, denoted $\operatorname{Comp}(\mathcal{S})$, is defined as $\operatorname{Comp}(M) \cap L$.

\subsection{DTL Specification of Reactive Systems}

As mentioned above, local properties are described by a machine and liveness properties as a set of histories. The dense temporal logic DTL, with syntax listed in table 1, describes both kinds of properties. To suit the main purpose of achieving a formalism for compositional refinement our logic is a carefully composed mixture of the dense temporal logics defined in [Sta84, BKP86, DK90, KMP93].

Table 1. Syntax of DTL. Let value $\mu \in V$ al, rigid variable $n \in \mathfrak{R}$, observable variable $\mathbf{v} \in \mathfrak{V}$, local variable $\mathrm{x} \in \mathfrak{X}$, event variable $\epsilon \in \mathfrak{E}$ and channel $\mathbf{a} \in$ Chan.

\begin{tabular}{|c|}
\hline \begin{tabular}{c}
\multicolumn{2}{c}{ Rigid Expressions } \\
$\operatorname{rexp}::=\mu|n| n^{\prime}\left|{ }^{\prime} n\right| \operatorname{rexp}_{1}+\operatorname{rexp}_{2} \mid \ldots$
\end{tabular} \\
\hline $\begin{array}{l}\text { Expressions } \\
\exp ::=\operatorname{rexp}|\mathbf{v}| \mathbf{v}^{\prime}|\mathbf{v}| \mathbf{x}\left|\mathbf{x}^{\prime}\right| \mathbf{x}\left|\exp p_{1}+\exp p_{2}\right| \ldots\end{array}$ \\
\hline $\begin{array}{l}\text { Event Expressions } \\
\operatorname{evexp}::=\left.\mathbf{a} ?|\mathbf{a} !| \mathbf{i}|\mathbf{e}| \lambda|\epsilon| \epsilon^{\prime}\right|^{`} \epsilon\end{array}$ \\
\hline $\begin{array}{l}\text { Temporal formulae } \\
p:=\text { true }\left|\exp _{1}=\exp _{2}\right| \exp _{1}<\exp _{2}\left|\operatorname{evexp}_{1}=\operatorname{evexp}_{2}\right| \neg p \\
p_{1} \vee p_{2}\left|p_{1} \widehat{\mathcal{U}} p_{2}\right| p_{1} \widehat{\mathcal{S}} p_{2} \mid \exists \text { x.p }|\exists \epsilon . p| \exists n . p\end{array}$ \\
\hline
\end{tabular}

The - and '-priming of a variable denotes respectively the previous and next value of the variable whereas the unprimed variable denotes the current value. $p_{1} \widehat{\mathcal{U}} p_{2}$ denotes strict (present not included in the future) until operator from temporal logic and $p_{1} \widehat{\mathcal{S}} p_{2}$ denotes strict (present not included in the past) 
since operator from temporal logic. $\exists$ x.p denotes existential quantification over local variable $\mathrm{x}$ of $p$, i.e., hiding. A state expression is an expression without any primed variables. A state formula is a formula build from state expressions without $\widehat{\mathcal{U}}$ and $\widehat{\mathcal{S}}$ operators. Table 2 lists some frequently used abbreviations:

Table 2. Frequently used abbreviations

\begin{tabular}{|c|c|c|}
\hline $\begin{array}{l}\widehat{\diamond} p \\
\widehat{\square} p \\
\bigcirc p \\
\diamond p \\
\square p \\
p_{1} \mathcal{U} p_{2} \\
\widehat{\ominus} p \\
\hat{\theta} p \\
\Theta p \\
\widetilde{\Theta} p \\
\text { first } \\
\ominus p \\
\boxminus p \\
p_{1} \mathcal{S} p_{2} \\
p_{1} \Rightarrow p_{2} \\
p_{1} \Leftrightarrow p_{2}\end{array}$ & $\begin{array}{l}\doteq \operatorname{true} \widehat{\mathcal{U}} p \\
\doteq \neg \widehat{\diamond} \neg p \\
\doteq p \widehat{\mathcal{U}} \text { true } \\
\doteq p \vee \widehat{\diamond} p \\
\doteq p \wedge \widehat{\square} p \\
\doteq p_{2} \vee\left(p_{1} \wedge\left(p_{1} \widehat{\mathcal{U}} p_{2}\right)\right) \\
\doteq \operatorname{true} \widehat{\mathcal{S}} p \\
\doteq \neg \widehat{\ominus} \neg p \\
\doteq p \widehat{\mathcal{S}} \text { true } \\
\doteq \neg \Theta \neg p \\
\doteq \widetilde{\Theta} \text { false } \\
\doteq p \vee \widehat{\ominus} p \\
\doteq p \wedge \widehat{\Xi} p \\
\doteq p_{2} \vee\left(p_{1} \wedge\left(p_{1} \widehat{\mathcal{S}} p_{2}\right)\right) \\
\doteq \square\left(p_{1} \rightarrow p_{2}\right) \\
\doteq \square\left(p_{1} \leftrightarrow p_{2}\right)\end{array}$ & $\begin{array}{l}\text { strict eventually } p, \\
\text { strict always } p, \\
\text { for some time in the future } p \text { holds without } \\
\text { interruption, } \\
\text { non-strict eventually, } \\
\text { non-strict always, } \\
\text { non-strict until, } \\
\text { strict once } p \text { in the past, } \\
\text { strict has-always-been } p, \\
\text { for some time in the past } p \text { held without } \\
\text { interruption, } \\
\text { recently } p \text { held, } \\
\text { first position in a history, } \\
\text { non-strict once in the past, } \\
\text { non-strict has-always-been, } \\
\text { non-strict since, } \\
p_{1} \text { entails } p_{2} \\
p_{1} \text { is congruent } p_{2}\end{array}$ \\
\hline
\end{tabular}

Example2 Some DTL formulae. $\left(\epsilon=\mathbf{a}_{0} \wedge \mathrm{x}=0 \wedge \mathrm{x}^{\prime}=1\right)$ (a state-transition), $\square \mathrm{x}>0$ (a safety property), and $\square(\mathrm{x}=0 \rightarrow \diamond \mathrm{x}>0)$ (a liveness property).

Before we give the semantics of DTL formulae we define for a variable $x$ (local or event) the $x$-variant of a history.

Definition 15 ( $x$-variant of a history) Let $h, h_{1} \in \mathcal{H}, w \in \mathfrak{X} \cup \Re$ and $\epsilon \in \mathfrak{E}$. $h_{1}$ is a w-variant of $h$ if $\psi_{1}=\psi$ and $\left.\theta_{1}\right|_{(\mathfrak{D} \cup \mathfrak{X} \cup \mathfrak{R}) \backslash\{w\}} ^{2}=\left.\theta\right|_{(\mathfrak{V} \cup \mathfrak{X} \cup \mathfrak{R}) \backslash\{w\}} ^{2}$. $h_{1}$ is a $\epsilon$-variant of $h$ if $\left.\psi_{1}\right|_{\mathfrak{E} \backslash\{\epsilon\}} ^{2}=\left.\psi\right|_{\mathfrak{E} \backslash\{\epsilon\}} ^{2}$ and $\theta_{1}=\theta$.

In the following definition the semantics of DTL is given without using valuation functions for expressions, i.e., this valuation function is implicitly defined by $\models$. By convention, boolean values are not explicitly denoted, i.e., we shall write $(h, t)=$ true rather than $(h, t) \models$ true $\doteq t$.

\section{Definition 16 (Semantics of DTL)}


Let $h=\langle\psi, \theta\rangle \in \mathcal{H}, t \in \mathbb{R}^{+}, n \in \mathfrak{R}, \mathbf{v} \in \mathfrak{V}, \mathrm{x} \in \mathfrak{X}$, and $\epsilon \in \mathfrak{E}$.

$$
\begin{array}{lll}
(h, t) \models \mu \doteq \mu & (h, t) \models \mathbf{a} ? \doteq \mathbf{a} ? & (h, t) \models \mathbf{a} ! \doteq \mathbf{a} ! \\
(h, t) \models \mathbf{i} \doteq \mathbf{i} & (h, t) \models \mathbf{e} \doteq \mathbf{e} & (h, t) \models \lambda \doteq \lambda \\
(h, t) \models n=\theta(0)(n) & (h, t) \models n^{\prime} \doteq \theta(0)(n) & (h, t) \models n \doteq \theta(0)(n) \\
(h, t) \models \mathrm{x} \doteq \theta(t)(\mathrm{x}) & (h, t) \models \mathbf{v} \doteq \theta(t)(\mathbf{v}) & (h, t) \models \epsilon \doteq \psi(t)(\epsilon) \\
(h, 0) \models \mathrm{x} \doteq \theta(0)(\mathrm{x}) & (h, 0) \models \mathbf{v} \doteq \theta(0)(\mathbf{v}) & (h, 0) \models \epsilon \doteq \psi(0)(\epsilon) \\
(h, t) \models \mathrm{x}^{\prime} \doteq \lim _{t \leftarrow t_{1}} \theta\left(t_{1}\right)(\mathrm{x}) & (h, t) \models \mathbf{v}^{\prime} \doteq \lim _{t \leftarrow t_{1}} \theta\left(t_{1}\right)(\mathbf{v}) & (h, t) \models \epsilon^{\prime} \doteq \lim _{t \leftarrow t_{1}} \psi\left(t_{1}\right)(\epsilon) \\
t>0 & & \\
(h, t) \models \mathrm{x} \doteq \lim _{t_{1} \rightarrow t} \theta\left(t_{1}\right)(\mathrm{x})(h, t) \models \mathbf{v} \doteq \lim _{t_{1} \rightarrow t} \theta\left(t_{1}\right)(\mathbf{v})(h, t) \models \epsilon \doteq \lim _{t_{1} \rightarrow t} \psi\left(t_{1}\right)(\epsilon) \\
(h, t) \models e x p_{1}+\exp _{2} \doteq(h, t) \models e x p_{1}+(h, t) \models e x p_{2} & \\
(h, t) \models e x p_{1}-\exp _{2} \doteq(h, t) \models e x p_{1}-(h, t) \models e x p_{2} & \\
(h, t) \models e x p_{1}=\exp _{2} \text { iff }(h, t) \models e x p_{1}=(h, t) \models e x p_{2} & \\
(h, t) \models e v e x p_{1}=e v e x p_{2} \text { iff }(h, t) \models e v e x p_{1}=(h, t) \models e v e x p_{2} \\
(h, t) \models e x p_{1}<\exp _{2} \text { iff }(h, t) \models e x p_{1}<(h, t) \models e x p_{2} & \\
(h, t) \models \operatorname{true} & &
\end{array}
$$

\section{Definition 17 (Satisfiability, validity)}

Let $p$ be a DTL formula, $h$ be a history, and $\mathcal{S}$ be a system with basis $B$.

$h$ satisfies $p$, denoted $h \models p$, iff $(h, 0) \mid=p$.

$p$ is satisfiable iff $h=p$ for some history $h \in \mathcal{H}$.

$p$ is valid, denoted $=p$, iff $h \models p$ for all histories $h \in \mathcal{H}$.

$p$ is $\mathcal{S}$-valid, denoted $\mathcal{S}=p$, iff $h=p$ for all histories $h \in \operatorname{Comp}(\mathcal{S})$.

Hist $(p)$ denotes the set of all histories satisfying $p$.

The following theorem states that the logic DTL is history process state stutter insensitive. Later on a restricted stutter insensitive version of DTL is considered.

\section{Theorem 1 (DTL is stutter insensitive)}

For rigid expression rexp, expression exp, event expression evexp and DTL formula $p$ :

$$
\begin{aligned}
& \forall t, h_{0}, h_{1}: h_{0} \simeq_{\theta_{h}} h_{1} \rightarrow\left(\left(h_{0}, t\right)=\exp =\left(h_{1}, \operatorname{di}\left(h_{1}\right) \circ \operatorname{di}^{-1}\left(h_{0}\right)(t)\right)=\exp \right) \\
& \forall t, h_{0}, h_{1}: h_{0} \simeq_{\theta_{h}} h_{1} \rightarrow\left(\left(h_{0}, t\right)=\exp =\left(h_{1}, \operatorname{di}\left(h_{1}\right) \circ \operatorname{di}^{-1}\left(h_{0}\right)(t)\right) \models \exp \right) \\
& \forall t, h_{0}, h_{1}: h_{0} \simeq_{\theta_{h}} h_{1} \rightarrow\left(\left(h_{0}, t\right)=\operatorname{evexp}=\left(h_{1}, \operatorname{di}\left(h_{1}\right) \circ \operatorname{di}^{-1}\left(h_{0}\right)(t)\right) \models \operatorname{evexp}\right) \\
& \forall t, h_{0}, h_{1}: h_{0} \simeq_{\theta_{h}} h_{1} \rightarrow\left(\left(h_{0}, t\right) \models p \text { iff }\left(h_{1}, \operatorname{di}\left(h_{1}\right) \circ \operatorname{di}^{-1}\left(h_{0}\right)(t)\right)=p\right)
\end{aligned}
$$

The proof system for DTL listed in tables 3 and 4 is inspired on [Bur82, Bur84, BKP86, MP89]. An erroneous variant of it appeared in [BKP86] $]^{4}$. Furthermore a link with the proof system of [KMP93] is established via axioms $A x 7 b-A x 7 f$,

\footnotetext{
${ }^{4}$ F5 was not copied correctly.
} 
i.e., since these axioms are needed for deriving their proof system. Note: the models of [Bur82, Bur84] need not to satisfy the finite variability condition, the persistency conditions (once within an interval "going a little bit back or forward" doesn't bring you outside that interval), and the induction axiom. This reflects the crucial difference between the model of [KMP93] and ours on the one side and the model of [Bur82, Bur84] on the other side. The difference between the model of [KMP93] and our model is that we introduced additional compositionality information reflected by axioms $A x 0, A x 5$ and $A x 6$.

The proof system is for the pure logic, i.e., it is not meant for a specific reactive system. Axioms $A x 0-A x 9$ characterise our notion of histories; they should follow from the definition of history (Def. 7), and, because a history is a pair consisting of a event and a state function, also from Definition 5 and 6 . Ax10 and $A x 11$ are the axioms for substitution and quantification. Axioms $F 1-F 7$ are the axioms of the future part of DTL and $P 1-P 7$ the past part. As rules we take standard ones, i.e., the modus ponus, generalisation, specialisation, instantiation and universal generalisation.

Table 3. Axioms. Let $n \in \mathfrak{R}, \mathrm{v} \in \mathfrak{V}, w \in \mathfrak{V} \cup \mathfrak{X}, \mathrm{x} \in \mathfrak{X}$ and $\epsilon \in \mathfrak{E}$.

S0: All the axioms for state formulae.

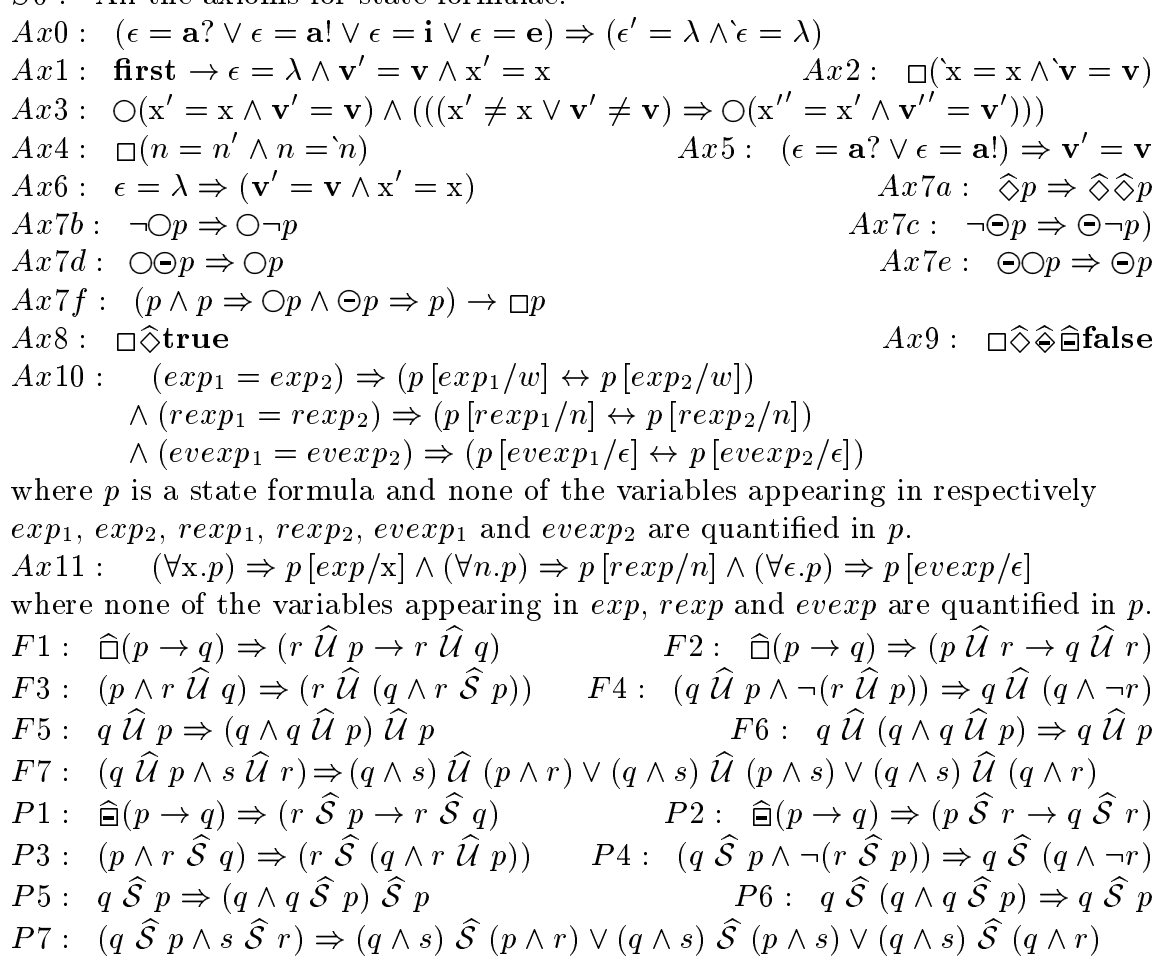


Table 4. Rules. Let $w \in \mathfrak{R} \cup \mathfrak{X} \cup \mathfrak{E}$.
$M P: \frac{p, p \rightarrow q}{q}$
$S P E C: \frac{\square p}{p}$ for state formula $p$
$G E N: \frac{p}{\widehat{\square} p}$ for state formula $\mathrm{p}$ in which all occurrences of
$I N S T: \frac{p}{p\left[p_{1} / p_{0}\right]}$ where $p_{1}$ doesn't contain variables which are bound in $p$
$U G E N-w: \frac{p_{0} \Rightarrow p_{1}}{p_{0} \Rightarrow \forall w \cdot p_{1}}$ for $w$ not free in $p_{0}$

The following definition characterises a machine $M$ in DTL. This kind of DTL formulae is history stutter insensitive.

Definition 18 (Machine in DTL) Given basis $B=((\mathrm{In}$, Out), (V, X)). Let In? be defined as $\{\mathbf{a}$ ? $\mid a \in \operatorname{In}\}$ and let Out! be defined as $\{\mathbf{a} ! \mid a \in$ Out $\}$. Let I be a DTL formula over $\mathrm{V} \cup \mathrm{X}$ without the $\widehat{\mathcal{S}}, \widehat{\mathcal{U}}$ and $\exists$ operators. Let $\mathcal{T}$ be a finite set of DTL formulae $\tau$ of the form $\left(\right.$ event $_{\tau} \wedge$ trans $_{\tau}$ ) where event w $_{\tau}$ is of the form $\epsilon=a_{\tau}$ where $a_{\tau} \in\{\mathbf{i}, \mathbf{e}\} \cup \operatorname{In}$ ? $\cup$ Out!, and trans $s_{\tau}$ a DTL formula over $\mathrm{V} \cup \mathrm{X}$ and $\mathrm{V}^{\prime} \cup \mathrm{X}^{\prime}$ (variables primed with') without the $\widehat{\mathcal{S}}, \widehat{\mathcal{U}}$ and $\exists$ operators such that $\left(\epsilon=\mathbf{e} \Rightarrow \bigwedge_{\mathrm{x} \in \mathrm{X}} \mathrm{x}^{\prime}=\mathrm{x}\right)$, i.e., an environment action doesn't change the local variables of the system. Define the stutter step, denoted by stut, as $\epsilon=\lambda \vee\left(\epsilon=\mathbf{i} \wedge(\mathrm{V}, \mathrm{X})^{\prime}=(\mathrm{V}, \mathrm{X})\right) \vee\left(\epsilon=\mathbf{e} \wedge(\mathrm{V}, \mathrm{X})^{\prime}=(\mathrm{V}, \mathrm{X})\right)$. Let $\mathrm{T}$ be the $D T L$ formula stut $\vee \bigvee_{\tau \in \mathcal{T}} \tau$. A machine in DTL is defined as $(B, \mathrm{I} \wedge \square \mathrm{T})$.

Lemma 2 Given a machine in $D T L(B, \mathrm{I} \wedge \square \mathrm{T})$ there exists a semantic machine $M=(B, I, T)$ such that $\operatorname{Comp}(M)=H i s t(\mathrm{I} \wedge \square \mathrm{T})$.

\section{Definition 19 (Machine specification of a system in DTL)}

Given a machine $(B, \mathrm{I} \wedge \square \mathrm{T})$ in DTL. Let $\mathrm{WF} \subseteq \mathcal{T}$ be the set of weak fair transitions and $\mathrm{SF} \subseteq \mathcal{T}$ be the set of strong fair transitions. For $\tau \in \mathcal{T}$ define the enabledness condition for $\tau$ denoted $\operatorname{En}(\tau)$ as $\exists \bar{v}_{0} . \tau\left[\bar{v}_{0} / \bar{v}^{\prime}\right]$ where $\tau\left[\bar{v}_{0} / \bar{v}^{\prime}\right]$ denotes the substitution of $\bar{v}_{0}$ (a list of variables not in $\mathrm{V} \cup \mathrm{X}$ ) for $\bar{v}^{\prime}$ (the list of primed variables in $\tau)$. Let $\mathrm{L}$ be the DTL formula $\bigwedge_{\tau \in \mathrm{WF}}(\diamond \square E n(\tau) \rightarrow \square \diamond \tau) \wedge$ $\bigwedge_{\tau \in \mathrm{SF}}(\square \diamond E n(\tau) \rightarrow \square \diamond \tau)$. The machine specification of a system in DTL is then a tuple $(B, \mathrm{I} \wedge \square \mathrm{T} \wedge \mathrm{L})$.

Note: in above definition, liveness property $\mathrm{L}$ is such that $\operatorname{cl}(\operatorname{Hist}(\mathrm{I} \wedge \square \mathrm{T}) \cap$ $H i s t(\mathrm{~L}))=H i s t(\mathrm{I} \wedge \square \mathrm{T})$, i.e., it satisfies the machine closedness property. With this proviso the following lemma becomes straight forward.

Lemma 3 Given DTL machine specification $(B, \mathrm{I} \wedge \square \mathrm{T} \wedge \mathrm{L})$ of a system, there exists a semantic machine specification $\mathcal{S}=(B, \operatorname{Comp}(M) \cap L)$ such that $\operatorname{Comp}(M) \cap$ $L=H i s t(\mathrm{I} \wedge \square \mathrm{T} \wedge \mathrm{L})$. 


\section{Refinement and Composition of Reactive System Specifications}

We introduce the notion of refinement and composition of reactive systems. Intuitively, refinement implies that the set of histories of a concrete system is a subset of the set of histories of the abstract system. Composition implies that the histories of the component systems are "merged" into composite histories, i.e., the histories of the composed system. Our merge operator is based on the merge operator of Aczel [Acz83]. Both are first defined at the semantic level and then for the DTL specifications.

\subsection{Semantic Refinement and Composition of Specifications}

Refinement and composition of reactive systems is defined at the semantical level. Refinement implies that the set of histories of a concrete system is a subset of the set of histories of an abstract system. Because histories also contain local information the subset relation doesn't correspond directly with refinement. The local information should first be projected away. The following definition captures this projection of local information.

Definition 20 (Observable system specification)

Given system specification $\mathcal{S}=(B, H)$ where $B=((\mathrm{In}, \mathrm{Out}),(\mathrm{V}, \mathrm{X}))$. The observable system specification is a pair $\left(\mathfrak{O}(B), \mathcal{O}_{\mathrm{X}}(H)\right)$ where $\mathfrak{O}(B)$ denotes the observable basis and is defined as $\mathfrak{O}(B) \doteq((\mathrm{In}$, Out $), \mathrm{V}, \emptyset)$ and $\mathcal{O}_{\mathrm{X}}(H)$ denotes the set of observable histories corresponding to $H$ and is defined as $\left\{h \in \mathcal{H} \mid \exists h_{1} \in H: h\right.$ is an X-variant of $\left.h_{1}\right\}$.

Definition 21 (Refinement of systems) Given concrete system $\mathcal{S}_{c} \doteq\left(B_{c}, H_{c}\right)$ and abstract system $\mathcal{S}_{a} \doteq\left(B_{a}, H_{a}\right)$. $\mathcal{S}_{c}$ refines $\mathcal{S}_{a}$, denoted $\mathcal{S}_{c}$ ref $\mathcal{S}_{a}$, iff $\mathfrak{O}\left(B_{c}\right)=$ $\mathfrak{O}\left(B_{a}\right)$ and $\mathcal{O}_{\mathrm{X}_{c}}\left(H_{c}\right) \subseteq \mathcal{O}_{\mathrm{X}_{a}}\left(H_{a}\right)$.

A more general definition of refinement would be one in which both the abstract and concrete system are composed of subsystems. Therefore the notion of composition is introduced. Intuitively the composition of two systems is that matching histories are merged into one history. The matching condition is illustrated in Figure 4, i.e., a history of one system matches a history of the other system if for all time points $t$ the following two conditions hold: (1) the state information of the two histories at time $t$ are same and also (2a) in both histories the $\lambda$-action occurs at time $t$ or $(2 \mathrm{~b})$ in both histories the environment action e occurs at time $t$ or (2c) in one history at time $t$ a process action $\mathbf{i}$ occurs and in the other one an environment action e occurs at time $t$ or $(2 \mathrm{~d})$ in both histories at time $t$ a communication action a occurs which is an input action in one of them and an output action in the other one, or (2e) in one history at time $t$ a communication action occurs which is not an communication action in the other one and in the other history an environment action e occurs. So it is prohibited that the two components each perform an $\mathbf{i}$ action simultaneously because we intend to model 
interleaving, where only communication actions can occur simultaneously. Two matching histories are then merged into one history by (1) "copying" the stateinformation of the two histories; in case (2a) the resulting event becomes $\lambda$; in case $(2 \mathrm{~b})$ the resulting event becomes e;in case (2c) the resulting event becomes $\mathbf{i} ;$ in case (2d) the resulting event becomes $\mathbf{i}$; and in case (2e) the resulting event becomes the communication action. See again Figure 4 for an illustration of this merging.

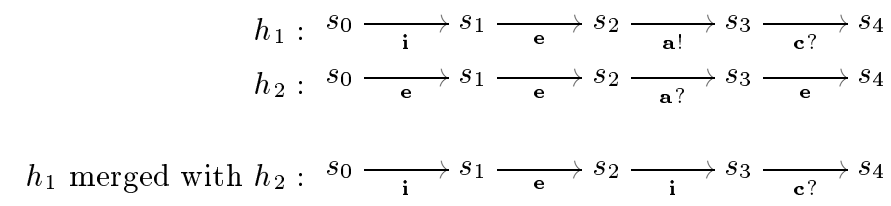

Fig. 4. Merging two matching histories.

Definition 22 (Composition of two systems) Given systems $\mathcal{S}_{i}=\left(B_{i}, H_{i}\right)$ with $B_{i}=\left(\left(\operatorname{In}_{i}\right.\right.$, Out $\left.\left._{i}\right),\left(\mathrm{V}_{i}, \mathrm{X}_{i}\right)\right)(i=1,2)$ such that $\operatorname{In}_{1} \cap \mathrm{In}_{2}=\emptyset$, Out ${ }_{1} \cap$ Out $_{2}=$ $\emptyset$ and $\mathrm{X}_{1} \cap \mathrm{X}_{2}=\emptyset$. The composed system $\mathcal{S}=\mathcal{S}_{1} \| \mathcal{S}_{2}$ is defined as $(B, H)$ with $B \doteq\left(\left(\mathrm{In}_{1} \backslash \mathrm{Out}_{2} \cup \mathrm{In}_{2} \backslash\right.\right.$ Out $_{1}$, Out $\left.\left._{1} \backslash \mathrm{In}_{2} \cup \mathrm{Out}_{2} \backslash \mathrm{In}_{1}\right),\left(\mathrm{V}_{1} \cup \mathrm{V}_{2}, \mathrm{X}_{1} \cup \mathrm{X}_{2}\right)\right)$ and $H \doteq H_{1} \otimes H_{2}$. The $\bigotimes$ symbol denotes a merge operator which merges the histories $h_{1} \in H_{1}$ and $h_{2} \in H_{2}$ into one history $h$ and is defined as $H_{1} \otimes H_{2} \doteq$ $\left\{h \in \mathcal{H} \mid \exists h_{1} \in H_{1}, h_{2} \in H_{2} \cdot \otimes\left(h, h_{1}, h_{2}\right)\right\}$ where for $h=\langle\psi, \theta\rangle$ and $h_{j}=\left\langle\psi_{j}, \theta_{j}\right\rangle$ $(j=1,2), \otimes\left(h, h_{1}, h_{2}\right)$ iff

$$
\begin{array}{ll}
1 & \theta=\theta_{1} \wedge \theta=\theta_{2} \\
2 & \forall t: \\
\vee & \psi(t)(\epsilon)=\lambda \wedge \psi_{1}(t)(\epsilon)=\lambda \wedge \psi_{2}(t)(\epsilon)=\lambda \\
\vee & \psi(t)(\epsilon)=\mathbf{e} \wedge \psi_{1}(t)(\epsilon)=\mathbf{e} \wedge \psi_{2}(t)(\epsilon)=\mathbf{e} \\
\vee & \psi(t)(\epsilon)=\mathbf{i} \wedge \psi_{1}(t)(\epsilon)=\mathbf{i} \wedge \psi_{2}(t)(\epsilon)=\mathbf{e} \\
\vee & \psi(t)(\epsilon)=\mathbf{i} \wedge \psi_{1}(t)(\epsilon)=\mathbf{e} \wedge \psi_{2}(t)(\epsilon)=\mathbf{i} \\
\vee & \exists \mathbf{a} \in \operatorname{In}_{1} \cap \operatorname{Out}_{2}: \psi(t)(\epsilon)=\mathbf{i} \wedge \psi_{1}(t)(\epsilon)=\mathbf{a} ? \wedge \psi_{2}(t)(\epsilon)=\mathbf{a} ! \\
\vee & \exists \mathbf{a} \in \operatorname{In}_{2} \cap \operatorname{Out}_{1}: \psi(t)(\epsilon)=\mathbf{i} \wedge \psi_{1}(t)(\epsilon)=\mathbf{a} ! \wedge \psi_{2}(t)(\epsilon)=\mathbf{a} ? \\
\vee & \exists \mathbf{a} \in \operatorname{In}_{1} \backslash \operatorname{Out}_{2}: \psi(t)(\epsilon)=\mathbf{a} ? \wedge \psi_{1}(t)(\epsilon)=\mathbf{a} ? \wedge \psi_{2}(t)(\epsilon)=\mathbf{e} \\
\vee & \exists \mathbf{a} \in \operatorname{Out}_{1} \backslash \operatorname{In}_{2}: \psi(t)(\epsilon)=\mathbf{a} ! \wedge \psi_{1}(t)(\epsilon)=\mathbf{a} ! \wedge \psi_{2}(t)(\epsilon)=\mathbf{e} \\
\vee & \exists \mathbf{a} \in \operatorname{In}_{2} \backslash \operatorname{Out}_{1}: \psi(t)(\epsilon)=\mathbf{a} ? \wedge \psi_{1}(t)(\epsilon)=\mathbf{e} \wedge \psi_{2}(t)(\epsilon)=\mathbf{a} ? \\
\vee & \exists \mathbf{a} \in \operatorname{Out}_{2} \backslash \operatorname{In}_{1}: \psi(t)(\epsilon)=\mathbf{a} ! \wedge \psi_{1}(t)(\epsilon)=\mathbf{e} \wedge \psi_{2}(t)(\epsilon)=\mathbf{a} !
\end{array}
$$

The following Lemma expresses that the "making observable"-operation and the merge operator are both monotonic and that the "making observable"-operation on the composed system is equal to the "making observable"-operation on the components. 
Lemma 4 (Properties of $\mathcal{O}$ and $\otimes$ )

Given systems $\left(B_{1}, H_{0}\right),\left(B_{1}, H_{1}\right),\left(B_{2}, H_{2}\right)$ and $\left(B_{2}, H_{3}\right)$ then

(a) $H_{0} \subseteq H_{1}$ implies $H_{0} \otimes H_{2} \subseteq H_{1} \otimes H_{2}$

(b) $\mathcal{O}_{\mathrm{X}_{12}}\left(H_{1} \otimes H_{2}\right)=\mathcal{O}_{\mathrm{X}_{1}}\left(H_{1}\right) \otimes \mathcal{O}_{\mathrm{X}_{2}}\left(H_{2}\right)$

(c) $H_{0} \subseteq H_{1}$ implies $\mathcal{O}_{\mathrm{X}_{1}}\left(H_{0}\right) \subseteq \mathcal{O}_{\mathrm{X}_{1}}\left(H_{1}\right)$

(d) $\left(H_{0} \cap H_{1}\right) \otimes\left(H_{2} \cap H_{3}\right) \subseteq\left(\bar{H}_{0} \otimes H_{2}\right) \cap\left(H_{1} \otimes H_{3}\right)$

Now the compositional refinement property can be inferred from the above lemma.

Theorem 2 (Compositional refinement) Given abstract systems $\mathcal{S}_{1}=\left(B_{1}, H_{1}\right)$ and $\mathcal{S}_{2}=\left(B_{2}, H_{2}\right)$, and given concrete systems $\mathcal{S}_{3}=\left(B_{3}, H_{3}\right)$ and $\mathcal{S}_{4}=\left(B_{4}, H_{4}\right)$ such that $\mathfrak{O}\left(B_{1}\right)=\mathfrak{O}\left(B_{3}\right)$ and $\mathfrak{O}\left(B_{2}\right)=\mathfrak{O}\left(B_{4}\right)$ then

$\mathcal{S}_{3}$ ref $\mathcal{S}_{1}$ and $\mathcal{S}_{4}$ ref $\mathcal{S}_{2}$ implies $\mathcal{S}_{3} \| \mathcal{S}_{4}$ ref $\mathcal{S}_{1} \| \mathcal{S}_{2}$

\subsection{Refinement and Composition of DTL Specifications}

The refinement and composition notion of the previous section are translated into DTL by defining this notion for machine specifications (Def. 19). This means that first the observable machine specification should be defined in DTL.

Definition 23 (Observable machine specification in DTL) Given machine specification $(B, \mathrm{I} \wedge \square \mathrm{T} \wedge \mathrm{L})$ in DTL, the corresponding observable machine specification is defined as $(\mathfrak{O}(B),(\exists \mathrm{X} .(\mathrm{I} \wedge \square \mathrm{T} \wedge \mathrm{L})))$.

The following lemma expresses that existential quantification relates to the semantic notion of observable histories.

Lemma 5 Given DTL machine specification $\mathcal{S}=(B, \mathrm{I} \wedge \square \mathrm{T} \wedge \mathrm{L})$ then $\mathcal{O}_{\mathrm{X}}(H i s t(\mathrm{I} \wedge$ $\square \mathrm{T} \wedge \mathrm{L}))=H i s t((\exists \mathrm{X} .(\mathrm{I} \wedge \square \mathrm{T} \wedge \mathrm{L})))$

Theorem 3 (Refinement of machine specifications) Given concrete machine specification $\mathcal{S}_{c} \doteq\left(B_{c}, \mathrm{I}_{c} \wedge \square \mathrm{T}_{c} \wedge \mathrm{L}_{c}\right)$ where $B_{c} \doteq\left(B_{c}^{A},\left(\mathrm{~V}_{c}, \mathrm{X}_{c}\right)\right)$ and abstract machine specification $\mathcal{S}_{a} \doteq\left(B_{a}, \mathrm{I}_{a} \wedge \square \mathrm{T}_{a} \wedge \mathrm{L}_{a}\right)$ where $B_{a} \doteq\left(B_{a}^{A},\left(\mathrm{~V}_{a}, \mathrm{X}_{a}\right)\right)$. Then $\mathcal{S}_{c}$ refines $\mathcal{S}_{a}$, denoted $\mathcal{S}_{c}$ ref $\mathcal{S}_{a}$, iff $\mathfrak{O}\left(B_{c}\right)=\mathfrak{O}\left(B_{a}\right)$ and $\left(\exists \mathrm{X}_{c} \cdot\left(\mathrm{I}_{c} \wedge \square \mathrm{T}_{c} \wedge \mathrm{L}_{c}\right)\right) \rightarrow$ $\left(\exists \mathrm{X}_{a} \cdot\left(\mathrm{I}_{a} \wedge \square \mathrm{T}_{a} \wedge \mathrm{L}_{a}\right)\right)$.

Composition of DTL machine specifications can be defined in the same way as in the previous section.

Definition 24 (Composition of two DTL machine specifications)

Given DTL machine system specifications $\mathcal{S}_{i} \doteq\left(B_{i}, \mathrm{I}_{i} \wedge \square \mathrm{T}_{i} \wedge \mathrm{L}_{i}\right)$ where $B_{i} \doteq$ 
$\left(B_{i}^{A}, B_{i}^{P}\right)$, for $i=1,2$. Let ${ }_{B_{1}^{A}} \odot_{B_{2}^{A}}\left(\epsilon, \epsilon_{1}, \epsilon_{2}\right)$ be defined as

$$
\begin{aligned}
& \square\left(\quad \epsilon=\lambda \wedge \epsilon_{1}=\lambda \wedge \epsilon_{2}=\lambda\right. \\
& \vee \epsilon=\mathbf{e} \wedge \epsilon_{1}=\mathbf{e} \wedge \epsilon_{2}=\mathbf{e} \\
& \vee \epsilon=\mathbf{i} \wedge \epsilon_{1}=\mathbf{i} \wedge \epsilon_{2}=\mathbf{e} \\
& \vee \epsilon=\mathbf{i} \wedge \epsilon_{1}=\mathbf{e} \wedge \epsilon_{2}=\mathbf{i} \\
& \vee \bigvee_{\mathbf{a} \in \operatorname{In}_{1} \cap \mathrm{Out}_{2}} \epsilon=\mathbf{i} \wedge \epsilon_{1}=\mathbf{a} ? \wedge \epsilon_{2}=\mathbf{a} \text { ! } \\
& \vee \bigvee_{\mathbf{a} \in \operatorname{In}_{2} \cap \mathrm{Out}_{1}} \epsilon=\mathbf{i} \wedge \epsilon_{1}=\mathbf{a} ! \wedge \epsilon_{2}=\mathbf{a} ? \\
& \vee \bigvee_{\mathbf{a} \in \mathrm{In}_{1} \backslash \mathrm{Out}_{2}} \epsilon=\mathbf{a} ? \wedge \epsilon_{1}=\mathbf{a} ? \wedge \epsilon_{2}=\mathbf{e} \\
& \vee \bigvee_{\mathbf{a} \in \mathrm{Out}_{1} \backslash \mathrm{In}_{2}} \epsilon=\mathbf{a} ! \wedge \epsilon_{1}=\mathbf{a} ! \wedge \epsilon_{2}=\mathbf{e} \\
& \vee \bigvee_{\mathbf{a} \in \mathrm{In}_{2} \backslash \mathrm{Out}} \epsilon=\mathbf{a} ? \wedge \epsilon_{1}=\mathbf{e} \wedge \epsilon_{2}=\mathbf{a} ? \\
& \left.\vee \bigvee_{\mathbf{a} \in \mathrm{Out}_{2} \backslash \mathrm{In}_{1}} \epsilon=\mathbf{a} ! \wedge \epsilon_{1}=\mathbf{e} \wedge \epsilon_{2}=\mathbf{a} !\right)
\end{aligned}
$$

Then the composed machine system specification $\mathcal{S}$ is defined as $(B, \mathrm{H})$ where

$$
\begin{aligned}
& \mathrm{H} \doteq \exists \epsilon_{1}, \epsilon_{2} \cdot B_{1}^{A} \odot_{B_{2}^{A}}\left(\epsilon, \epsilon_{1}, \epsilon_{2}\right) \wedge\left(\mathrm{I}_{1} \wedge \square \mathrm{T}_{1} \wedge \mathrm{L}_{1}\right)\left[\epsilon_{1} / \epsilon\right] \wedge\left(\mathrm{I}_{2} \wedge \square \mathrm{T}_{2} \wedge \mathrm{L}_{2}\right)\left[\epsilon_{2} / \epsilon\right] \\
& B \doteq\left(\left(\mathrm{In}_{1} \backslash \text { Out }_{2} \cup \mathrm{In}_{2} \backslash \text { Out }_{1}, \text { Out }_{1} \backslash \mathrm{In}_{2} \cup \mathrm{Out}_{2} \backslash \mathrm{In}_{1}\right),\left(\mathrm{V}_{1} \cup \mathrm{V}_{2}, \mathrm{X}_{1} \cup \mathrm{X}_{2}\right)\right)
\end{aligned}
$$

This definition can be easily extended for $n$ DTL specifications. One has only to define a predicate $\odot_{\bar{B}^{A}}(\epsilon, \bar{\epsilon})$ corresponding to the operation of merging $n$ components.

Theorem 4 (Semantic merge is almost conjunction) Given the component machine system specifications $\mathcal{S}_{1}$ and $\mathcal{S}_{2}$, and the composed machine system specification $\mathcal{S}$ of definition 24 then $\operatorname{Hist}\left(\mathrm{I}_{1} \wedge \square \mathrm{T}_{1} \wedge \mathrm{L}_{1}\right) \otimes \operatorname{Hist}\left(\mathrm{I}_{2} \wedge \square \mathrm{T}_{2} \wedge \mathrm{L}_{2}\right)=$ $\operatorname{Hist}(\mathrm{H})$.

Example 3.

Given systems $\mathcal{S}_{1} \doteq\left(B_{1}, \mathrm{I}_{1} \wedge \square \mathrm{T}_{1} \wedge \mathrm{L}_{1}\right)$ and $\mathcal{S}_{2} \doteq\left(B_{2}, \mathrm{I}_{2} \wedge \square \mathrm{T}_{2} \wedge \mathrm{L}_{2}\right)$ where

$$
\begin{aligned}
B_{1} \doteq & ((\{\mathbf{c}\},\{\mathbf{a}\}),(\{\mathbf{s}\},\{\mathrm{t}\})), \mathrm{I}_{1} \doteq(\mathbf{s}, \mathrm{t})=(0,0), \mathrm{L}_{1} \doteq \mathbf{t r u e} \\
\mathrm{T}_{1} \doteq & \left(\epsilon=\mathbf{a} ! \wedge \mathrm{t}=0 \wedge(\mathbf{s}, \mathrm{t})^{\prime}=(\mathbf{s}, 1)\right) \vee\left(\epsilon=\mathbf{c} ? \wedge \mathrm{t}=1 \wedge(\mathbf{s}, \mathrm{t})^{\prime}=(\mathbf{s}, 2)\right) \vee \\
& \left(\epsilon=\mathbf{e} \wedge(\mathbf{s}, \mathrm{t})^{\prime}=(1, \mathrm{t})\right) \vee\left(\epsilon=\mathbf{e} \wedge(\mathbf{s}, \mathrm{t})^{\prime}=(0, \mathrm{t})\right) \vee \mathbf{s t u t}_{1} \\
B_{2} \doteq & ((\{\mathbf{b}\},\{\mathbf{c}\}),(\{\mathbf{s}\},\{\mathrm{u}\})), \mathrm{I}_{2} \doteq(\mathbf{s}, \mathrm{u})=(0,0), \mathrm{L}_{2} \doteq \mathbf{t r u e} \\
\mathrm{T}_{2} \doteq & \left(\epsilon=\mathbf{c} ! \wedge \mathrm{u}=0 \wedge(\mathbf{s}, \mathrm{u})^{\prime}=(\mathbf{s}, 1)\right) \vee\left(\epsilon=\mathbf{b} ? \wedge \mathrm{u}=1 \wedge(\mathbf{s}, \mathrm{u})^{\prime}=(\mathbf{s}, 2)\right) \vee \\
& \left(\epsilon=\mathbf{i} \wedge(\mathbf{s}, \mathrm{u})=(1,2) \wedge(\mathbf{s}, \mathrm{u})^{\prime}=(0, \mathrm{u})\right) \vee\left(\epsilon=\mathbf{e} \wedge(\mathbf{s}, \mathrm{u})^{\prime}=(1, \mathrm{u})\right) \vee \mathbf{s t u t}_{2}
\end{aligned}
$$

The transitions of machine 1 and 2 are illustrated in figure 5 . Note: the stutter transitions are not drawn in all subsequent figures in order to minimise the number of edges.

According to definition 24 the composition of $\mathcal{S}_{c_{1}}$ and $\mathcal{S}_{c_{2}}$ is as follows:

$$
\begin{aligned}
& (((\{\mathbf{a}\},\{\mathbf{b}\}),(\{\mathbf{s}\},\{\mathrm{t}, \mathrm{u}\})), \\
& \left.\quad \exists \epsilon_{1}, \epsilon_{2} \cdot\left(B_{1}^{A} \odot_{B_{2}^{A}}^{A}\left(\epsilon, \epsilon_{1}, \epsilon_{2}\right) \wedge\left(\mathrm{I}_{1} \wedge \square \mathrm{T}_{1} \wedge \mathrm{L}_{1}\right)\left[\epsilon_{1} / \epsilon\right] \wedge\left(\mathrm{I}_{2} \wedge \square \mathrm{T}_{2} \wedge \mathrm{L}_{2}\right)\left[\epsilon_{2} / \epsilon\right]\right)\right)
\end{aligned}
$$



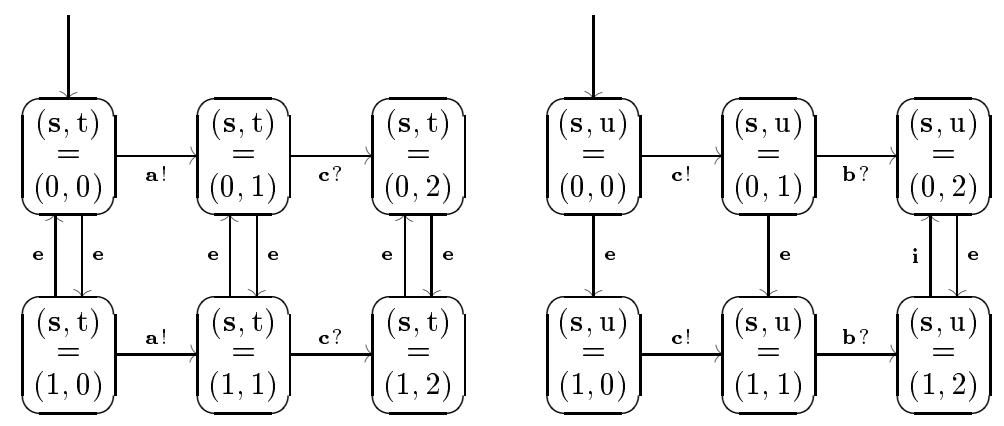

Fig. 5. Transitions of $\mathcal{S}_{1}$ and $\mathcal{S}_{2}$.

\section{Proving Refinement of Reactive System Specifications}

This section explains how refinement of reactive systems can be proved. The standard technique of Abadi and Lamport [AL91] is used, i.e., refinement is proven by providing a refinement mapping from the concrete system to the abstract system. First we give its definition at the semantic level and then for DTL specifications.

\subsection{Proving Semantic Refinement of Specifications}

Refinement of reactive systems is proved by means of a refinement mapping from the concrete system to the abstract system. A refinement mapping maps a history at the concrete level to a history at the abstract level, more specifically, it maps the states appearing in the concrete history to states appearing in the abstract history.

Definition 25 (Systems refinement mapping) Given concrete system $\mathcal{S}_{c} \doteq$ $\left(B_{c}, H_{c}\right)$ and abstract system $\mathcal{S}_{a} \doteq\left(B_{a}, H_{a}\right)$ s.t. $\mathfrak{O}\left(B_{c}\right)=\mathfrak{O}\left(B_{a}\right)$. A refinement mapping from $\mathcal{S}_{c}$ to $\mathcal{S}_{a}$ is a mapping $f$ from states appearing in histories of $H_{c}$ to states appearing in histories of $H_{a}$, i.e., $f: \Sigma \rightarrow \Sigma$ s.t. (1) the values of observable variables are not changed, i.e., for all $\sigma \in \Sigma:\left.\sigma\right|_{\mathrm{V}_{c}} ^{1}=\left.f(\sigma)\right|_{\mathrm{V}_{c}} ^{1}$, and (2) for all $h_{c} \in H_{c}$ there exists a $h_{a} \in H_{a}$ s.t. for all $t \in \mathbb{R}^{+}, \psi_{c}(t)=\psi_{a}(t)$ and $\theta_{a}(t)=f\left(\theta_{c}(t)\right)$.

Lemma 6 Given concrete system $\mathcal{S}_{c} \doteq\left(B_{c}, H_{c}\right)$ and abstract system $\mathcal{S}_{a} \doteq$ $\left(B_{a}, H_{a}\right)$ s.t. $\mathfrak{O}\left(B_{c}\right)=\mathfrak{O}\left(B_{a}\right)$. If there exists a refinement mapping from $\mathcal{S}_{c}$ to $\mathcal{S}_{a}$, then $\mathcal{S}_{c}$ ref $\mathcal{S}_{a}$.

Next the concept of refinement mappings is applied to machine specifications. Refinement means then that $f\left(\operatorname{Comp}\left(M_{c}\right) \cap L_{c}\right) \subseteq \operatorname{Comp}\left(M_{a}\right) \cap L_{a}$ for refinement mapping $f$. This can be split into (1) $f\left(\operatorname{Comp}\left(M_{c}\right) \cap L_{c}\right) \subseteq \operatorname{Comp}\left(M_{a}\right)$ and 
(2) $f\left(\operatorname{Comp}\left(M_{c}\right) \cap L_{c}\right) \subseteq L_{a}$. From $f\left(\operatorname{Comp}\left(M_{c}\right)\right) \subseteq \operatorname{Comp}\left(M_{a}\right)$ follows (1) because $f\left(\operatorname{Comp}\left(M_{c}\right) \cap \bar{L}_{c}\right) \subseteq f\left(\operatorname{Comp}\left(M_{c}\right)\right)$. So the verification condition can be split into a condition on machines and a condition involving machines and supplementary conditions. This leads to the following definition.

Definition 26 (Machine specification refinement mapping) Given abstract machine specification $\mathcal{S}_{a} \doteq\left(B_{a}, \operatorname{Comp}\left(M_{a}\right) \cap L_{a}\right)$, where $M_{a} \doteq\left(B_{a}, I_{a}, T_{a}\right)$, and concrete machine specification $\mathcal{S}_{c} \doteq\left(B_{c}, \operatorname{Comp}\left(M_{c}\right) \cap L_{c}\right)$, where $M_{c} \doteq$ $\left(B_{c}, I_{c}, T_{c}\right)$.

$A$ refinement mapping from $\mathcal{S}_{c}$ to $\mathcal{S}_{a}$ is a mapping $f: \Sigma \rightarrow \Sigma$ s.t.

(1) $\forall \sigma \in \Sigma .\left.\sigma\right|_{\mathrm{V}_{c}} ^{1}=\left.f(\sigma)\right|_{\mathrm{V}_{c}} ^{1}$,

(2a) $\forall \sigma_{c} \in I_{c} . \exists \sigma_{a} \in I_{a} \cdot \sigma_{a}=f\left(\sigma_{c}\right)$,

(2b) $\forall\left\langle d, \sigma_{c 1}, \sigma_{c 2}\right\rangle \in T_{c} .\left\langle d, f\left(\sigma_{c 1}\right), f\left(\sigma_{c 2}\right)\right\rangle \in T_{a} \vee\left(f\left(\sigma_{c 1}\right)=f\left(\sigma_{c 2}\right) \wedge d(\epsilon) \in\right.$ $\{\lambda, \mathbf{i}, \mathbf{e}\}$,

(2c) $\forall h_{c} \in \operatorname{Comp}\left(M_{c}\right) \cap L_{c} . \exists h_{a} \in L_{a} . \forall t \in \mathbb{R}^{+} . \psi_{c}(t)=\psi_{a}(t) \wedge f\left(\theta_{c}(t)\right)=$ $\theta_{a}(t)$.

That refinement mappings are indeed sound for proving refinement of machine specifications, is stated in:

Lemma 7 Given concrete machine specification $\mathcal{S}_{c} \doteq\left(B_{c}, \operatorname{Comp}\left(M_{c}\right) \cap L_{c}\right)$ and abstract machine specification $\mathcal{S}_{a} \doteq\left(B_{a}, \operatorname{Comp}\left(M_{a}\right) \cap L_{a}\right)$ s.t. $\mathfrak{O}\left(B_{c}\right)=\mathfrak{O}\left(B_{a}\right)$. If there exists a refinement mapping from $\mathcal{S}_{c}$ to $\mathcal{S}_{a}$ then $\mathcal{S}_{c}$ ref $\mathcal{S}_{a}$.

\subsection{Proving Refinement of DTL Specifications}

Proving refinement of machine specifications in DTL amounts according to Theorem 3 to (1) the observable bases are equal and (2) that a certain formula with two existential quantifications is valid. More specifically:

Definition 27 (Refinement of DTL machine specifications) Given concrete machine specification $\mathcal{S}_{c} \doteq\left(B_{c}, \mathrm{I}_{c} \wedge \square \mathrm{T}_{c} \wedge \mathrm{L}_{c}\right)$ and abstract machine specification $\mathcal{S}_{a} \doteq\left(B_{a}, \mathrm{I}_{a} \wedge \square \mathrm{T}_{a} \wedge \mathrm{L}_{a}\right)$. Then $\mathcal{S}_{c}$ refines $\mathcal{S}_{a}$, denoted $\mathcal{S}_{c}$ ref $\mathcal{S}_{a}$, is defined by $\mathfrak{O}\left(B_{c}\right)=\mathfrak{O}\left(B_{a}\right)$ and $\left(\exists \mathrm{X}_{c} .\left(\mathrm{I}_{c} \wedge \square \mathrm{T}_{c} \wedge \mathrm{L}_{c}\right)\right) \rightarrow\left(\exists \mathrm{X}_{a} .\left(\mathrm{I}_{a} \wedge \square \mathrm{T}_{a} \wedge \mathrm{L}_{a}\right)\right)$.

So we must have a rule to prove the following implication: $\exists \mathrm{x}_{0} \cdot p_{0} \rightarrow \exists \mathrm{x}_{1} \cdot p_{1}$. The following rule, derived from the proof system of table 3 and 4 , does the job:

$$
\frac{p_{0} \rightarrow p_{1}\left[\exp / \mathrm{x}_{1}\right]}{\exists \mathrm{x}_{0} \cdot p_{0} \rightarrow \exists \mathrm{x}_{1} \cdot p_{1}} \begin{aligned}
& \text { for } \mathrm{x}_{0} \text { not free in } p_{1} \text { and none of the variables } \\
& \text { appearing in } \exp \text { is quantified in } p_{1}
\end{aligned}
$$

From the previous section it should be clear that exp expresses exactly the refinement mapping $f$, and that the proof can be split in a safety part and a liveness part (i.e., the proof of $p_{0} \rightarrow p_{1}\left[\exp / \mathrm{x}_{1}\right]$ of above rule is split into a safety and a liveness part). This culminates in the following proof rule for refinement based on similar ones in [Lam94, KMP93]. 


\section{Rule 1 (Proof rule for refinement)}

Given concrete machine specification $\mathcal{S}_{c} \doteq\left(B_{c}, \mathrm{I}_{c} \wedge \square \mathrm{T}_{c} \wedge \mathrm{L}_{c}\right)$ and abstract machine specification $\mathcal{S}_{a} \doteq\left(B_{a}, \mathrm{I}_{a} \wedge \square \mathrm{T}_{a} \wedge \mathrm{L}_{a}\right)$ s.t. $\mathfrak{O}\left(B_{c}\right)=\mathfrak{O}\left(B_{a}\right)$. Let $f$ be a refinement mapping from $\mathcal{S}_{c}$ to $\mathcal{S}_{a}$ then

$$
\frac{\mathcal{S}_{c} \models \mathrm{I}_{c} \rightarrow \mathrm{I}_{a}\left[f / \mathrm{X}_{a}\right], \quad \mathcal{S}_{c}=\mathrm{T}_{c} \rightarrow \mathrm{T}_{a}\left[f / \mathrm{X}_{a}\right], \mathcal{S}_{c}=\mathrm{L}_{a}\left[f / \mathrm{X}_{a}\right]}{=\left(\exists \mathrm{X}_{c} \cdot\left(\mathrm{I}_{c} \wedge \square \mathrm{T}_{c} \wedge \mathrm{L}_{c}\right)\right) \rightarrow\left(\exists \mathrm{X}_{a} \cdot\left(\mathrm{I}_{a} \wedge \square \mathrm{T}_{a} \wedge \mathrm{L}_{a}\right)\right)}
$$

Rule 1 and Theorem 2 are used in the following example.

Example 4.

As abstract machine specification we take the composition of the machine specifications $\mathcal{S}_{1}$ and $\mathcal{S}_{2}$ of example 3 . As concrete machine specification we take the composition of following machine specifications $\mathcal{S}_{3} \doteq\left(B_{3}, \mathrm{I}_{3} \wedge \square \mathrm{T}_{3} \wedge \mathrm{L}_{3}\right)$ and again $\mathcal{S}_{2}$ where

$$
\begin{aligned}
B_{3} \doteq & ((\{\mathbf{c}\},\{\mathbf{a}\}),(\{\mathbf{s}\},\{\mathrm{v}\})), \mathrm{I}_{3} \doteq(\mathbf{s}, \mathrm{v})=(0,0), \mathrm{L}_{3} \doteq \text { true } \\
\mathrm{T}_{3} \doteq & \left(\epsilon=\mathbf{a} ! \wedge \mathrm{v}=0 \wedge(\mathbf{s}, \mathrm{v})^{\prime}=(\mathbf{s}, 1)\right) \vee\left(\epsilon=\mathbf{i} \wedge \mathrm{v}=1 \wedge(\mathbf{s}, \mathrm{v})^{\prime}=(\mathbf{s}, 2)\right) \vee \\
& \left(\epsilon=\mathbf{c} ? \wedge \mathrm{v}=2 \wedge(\mathbf{s}, \mathrm{v})^{\prime}=(\mathbf{s}, 3)\right) \vee\left(\epsilon=\mathbf{e} \wedge(\mathbf{s}, \mathrm{v})^{\prime}=(1, \mathrm{v})\right) \vee \\
& \left(\epsilon=\mathbf{e} \wedge(\mathbf{s}, \mathrm{v})^{\prime}=(0, \mathrm{v})\right) \vee \mathbf{s t u t}_{3}
\end{aligned}
$$

So $\mathcal{S}_{3}$ differs from $\mathcal{S}_{1}$ in that it has an extra internal step before the $\mathbf{c}$ ? communication.

We want to prove $\mathcal{S}_{3} \| \mathcal{S}_{2}$ ref $\mathcal{S}_{1} \| \mathcal{S}_{2}$. Instead of constructing the two compositions and then applying Rule 1, we will first use Theorem 2 and then Rule 1 . That is, we only have to prove that (1) $\mathcal{S}_{3}$ ref $\mathcal{S}_{1}$ and $(2) \mathcal{S}_{2}$ ref $\mathcal{S}_{2}$. Case (2) is trivial, so we will show the proof of (1). The condition that the observable bases are equal is trivial. So we must prove

$$
\left(\exists \mathrm{X}_{3} \cdot\left(\mathrm{I}_{3} \wedge \square \mathrm{T}_{3} \wedge \mathrm{L}_{3}\right)\right) \rightarrow\left(\exists \mathrm{X}_{1} \cdot\left(\mathrm{I}_{1} \wedge \square \mathrm{T}_{1} \wedge \mathrm{L}_{1}\right)\right)
$$

This will be proven with Rule 1 . This means one has to find a refinement mapping $f$. In order to find such a mapping the transitions of $\mathcal{S}_{3}$ and $\mathcal{S}_{1}$ are illustrated in fig. 6 .

From fig. 6 one sees that $f$ should be defined as follows:

$$
\text { if } \begin{aligned}
\mathrm{v} & =0 \vee \mathrm{v}=1 \text { then } f(\mathrm{~s}, \mathrm{v})=\mathrm{v} \\
\mathrm{v} & =2 \vee \mathrm{v}=3 \text { then } f(\mathrm{~s}, \mathrm{v})=\mathrm{v}-1 f i
\end{aligned}
$$

The following premises should be valid in order to apply the rule:

$-\mathcal{S}_{3}=(\mathbf{s}, \mathrm{v})=(0,0) \rightarrow((\mathbf{s}, \mathrm{t})=(0,0))[f / \mathrm{t}]$. Applying substitution results in $(\mathbf{s}, \mathrm{v})=(0,0) \rightarrow(\mathbf{s}, \mathrm{v})=(0,0)$ and this is valid.

$-\mathcal{S}_{3}=\left(\epsilon=\mathbf{a} ! \wedge \mathrm{v}=0 \wedge(\mathbf{s}, \mathrm{v})^{\prime}=(\mathbf{s}, 1)\right) \rightarrow\left(\epsilon=\mathbf{a} ! \wedge \mathrm{t}=0 \wedge(\mathbf{s}, \mathrm{t})^{\prime}=(\mathbf{s}, 1)\right)[f / \mathrm{t}]$. Applying substitution results in: $\mathcal{S}_{3}=\left(\epsilon=\mathbf{a} ! \wedge \mathrm{v}=0 \wedge(\mathbf{s}, \mathrm{v})^{\prime}=(\mathbf{s}, 1)\right) \rightarrow$ $\left(\epsilon=\mathbf{a} ! \wedge \mathrm{v}=0 \wedge(\mathbf{s}, \mathrm{v})^{\prime}=(\mathbf{s}, 1)\right)$ and this is valid.

$-\mathcal{S}_{3}=\left(\epsilon=\mathbf{i} \wedge \mathrm{v}=1 \wedge(\mathbf{s}, \mathrm{v})^{\prime}=(\mathbf{s}, 2)\right) \rightarrow \mathbf{s t u t}_{1}[f / \mathrm{t}]$. Because of definition of stut $_{1}$ it suffices to prove: $\mathcal{S}_{3}=\left(\epsilon=\mathbf{i} \wedge \mathrm{v}=1 \wedge(\mathbf{s}, \mathrm{v})^{\prime}=(\mathbf{s}, 2)\right) \rightarrow(\epsilon=$ $\left.\mathbf{i} \wedge(\mathbf{s}, \mathrm{t})^{\prime}=(\mathbf{s}, \mathrm{t})\right)[f / \mathrm{t}]$. Applying substitution results in: $\mathcal{S}_{c} \models(\epsilon=\mathbf{i} \wedge \mathrm{v}=$ $\left.1 \wedge(\mathbf{s}, \mathrm{v})^{\prime}=(\mathrm{s}, 2)\right) \rightarrow\left(\epsilon=\mathbf{i} \wedge(\mathbf{s}, \mathrm{v}-1)^{\prime}=(\mathrm{s}, \mathrm{v})\right)$ and this is valid. 

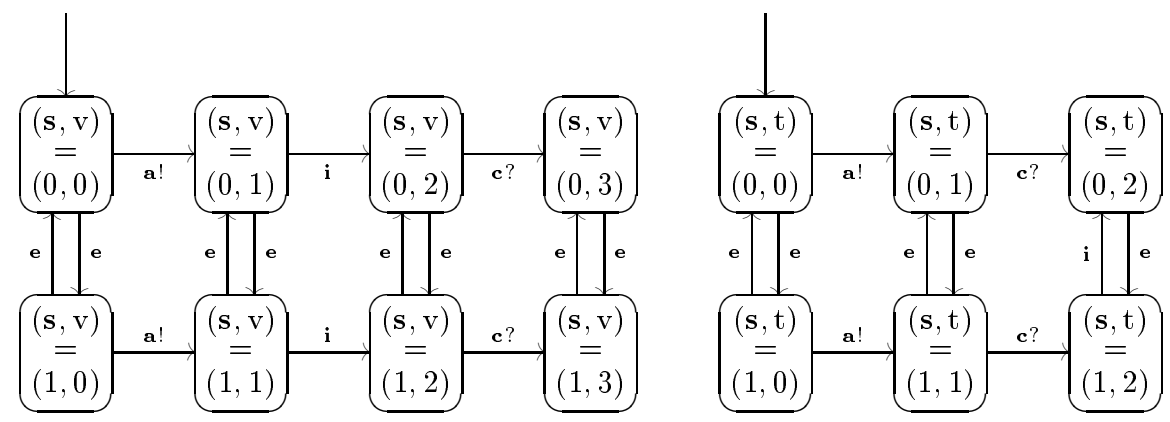

Fig. 6. Transitions of concrete $\mathcal{S}_{3}$ and abstract $\mathcal{S}_{1}$.

$-\mathcal{S}_{3} \models\left(\epsilon=\mathbf{c} ? \wedge \mathrm{v}=2 \wedge(\mathbf{s}, \mathrm{v})^{\prime}=(\mathbf{s}, 3)\right) \rightarrow\left(\epsilon=\mathbf{c} ? \wedge \mathrm{t}=1 \wedge(\mathbf{s}, \mathrm{t})^{\prime}=(\mathbf{s}, 2)\right)[f / \mathrm{t}]$. Applying substitution results in: $\mathcal{S}_{3}=\left(\epsilon=\mathbf{c} ? \wedge \mathrm{v}=2 \wedge(\mathbf{s}, \mathrm{v})^{\prime}=(\mathbf{s}, 3)\right) \rightarrow$ $\left(\epsilon=\mathbf{c} ? \wedge \mathrm{v}-1=1 \wedge(\mathbf{s}, \mathrm{v}-1)^{\prime}=(\mathbf{s}, 2)\right)$ and this is valid.

$-\mathcal{S}_{3} \models\left(\epsilon=\mathbf{e} \wedge(\mathrm{s}, \mathrm{v})^{\prime}=(1, \mathrm{v})\right) \rightarrow\left(\epsilon=\mathbf{e} \wedge(\mathrm{s}, \mathrm{t})^{\prime}=(1, \mathrm{t})\right)[f / \mathrm{t}]$. Applying substitution results in: $\mathcal{S}_{3}=\left(\epsilon=\mathbf{e} \wedge(\mathbf{s}, \mathrm{v})^{\prime}=(1, \mathrm{v})\right) \rightarrow\left(\epsilon=\mathbf{e} \wedge(\mathbf{s}, f)^{\prime}=\right.$ $(1, f))$ and this is valid because of $\mathrm{v}^{\prime}=\mathrm{v} \Rightarrow f^{\prime}=f$.

$-\mathcal{S}_{3} \models\left(\epsilon=\mathbf{e} \wedge(\mathbf{s}, \mathrm{v})^{\prime}=(0, \mathrm{v})\right) \rightarrow\left(\epsilon=\mathbf{e} \wedge(\mathbf{s}, \mathrm{t})^{\prime}=(0, \mathrm{t})\right)[f / \mathrm{t}]$. Applying substitution results in: $\mathcal{S}_{3}=\left(\epsilon=\mathbf{e} \wedge(\mathbf{s}, \mathrm{v})^{\prime}=(0, \mathrm{v})\right) \rightarrow\left(\epsilon=\mathbf{e} \wedge(\mathbf{s}, f)^{\prime}=\right.$ $(0, f))$ and this is valid because of $\mathrm{v}^{\prime}=\mathrm{v} \Rightarrow f^{\prime}=f$.

- $\mathcal{S}_{3}=$ stut $_{3} \rightarrow$ stut $_{1}[f / \mathrm{t}]$. Definition of stut $_{3}$ and stut ${ }_{1}$ results in $\mathcal{S}_{2} \models$ $\epsilon=\lambda \vee\left(\epsilon=\mathbf{i} \wedge(\mathbf{s}, \mathrm{v})^{\prime}=(\mathbf{s}, \mathrm{v})\right) \vee\left(\epsilon=\mathbf{e} \wedge(\mathbf{s}, \mathrm{v})^{\prime}=(\mathbf{s}, \mathrm{v})\right) \rightarrow \epsilon=\lambda \vee(\epsilon=$ $\left.\mathbf{i} \wedge(\mathbf{s}, \mathrm{t})^{\prime}=(\mathbf{s}, \mathrm{t})\right)[f / \mathrm{t}] \vee\left(\epsilon=\mathbf{e} \wedge(\mathbf{s}, \mathrm{t})^{\prime}=(\mathbf{s}, \mathrm{t})\right)[f / \mathrm{t}]$ and this is valid.

$-\mathcal{S}_{c}=$ true $[f / \mathrm{t}]$. This is valid.

\section{Conclusion}

It is shown that a dense temporal can be devised in which you can express compositionality and refinement of reactive systems. Furthermore a compositional refinement rule can be derived which enables ones to to prove refinement of large reactive systems from proofs of refinement between their components. In [Cau95] this rule is extensively used to prove the correctness of an implementation of stable storage and the correctness of Dijkstra's solution to the readers/writers synchronisation problem.

\section{Acknowledgements}

This work would not be finished without the help of Pierre Collette, Ruurd Kuiper, Yassine Lakhneche, Qiwen Xu and Kai Engelhardt, who all made many helpful comments. 


\section{References}

[Acz83] P. Aczel. On an inference rule for parallel composition, 1983. Unpublished, University of Manchester.

[AFK88] K. R. Apt, N. Francez, and S. Katz. Appraising fairness in languages for distributed programming. Distributed Computing, 2(4):226-241, 1988.

[AL91] M. Abadi and L. Lamport. The existence of refinement mappings. Theoretical Computer Science, 82(2):253-284, May 1991.

[AS85] B. Alpern and F.B. Schneider. Defining liveness. Information Processing Letters, 21(4):181-185, 1985.

[AS87] B. Alpern and F. Schneider. Proving boolean combinations of deterministic properties. In Proceedings of the second symposium on logic in computer science, pages 131-137. IEEE, June 1987.

[BKP84] H. Barringer, R. Kuiper, and A. Pnueli. Now you may compose temporal logic specifications. In Proceedings of 16th ACM Symposium on Theory of Computing, pages 51-63, 1984.

[BKP86] H. Barringer, R. Kuiper, and A. Pnueli. A really abstract concurrent model and its temporal semantics. In Proc. 13th ACM Symp. Princ. of Prog. Lang., pages 173-183, 1986.

[Bur82] J.P. Burgess. Axioms for tense logic, i. "since" and "until". Notre Dame Journal of Formal Logic, 23(4):367-374, October 1982.

[Bur84] J.P. Burgess. Basic tense logic. In D. Gabbay and F. Guenthner, editors, Handbook of Philosophical Logic., volume II, pages 89-133. Reidel Publishers, 1984.

[Cau95] A. Cau. Compositional Verification and Specification of Refinement for Reactive Systems in Dense Time Temporal Logic. PhD thesis, ChristianAlbrechts-Universität zu Kiel, 1995. Available as report no. 9601.

[CC96] A. Cau and P. Collette. Parallel composition of assumption-commitment specifications: a unifying approach for shared variable and distributed message passing concurrency. Acta Informatica, 33(2):153-176, 1996.

[DK90] E. Diepstraten and R. Kuiper. Abadi \& Lamport and Stark: towards a proof theory for stuttering, dense domains and refinements mappings. In LNCS 430:Proc. of the REX Workshop on Stepwise Refinement of Distributed Systems, Models, Formalisms, Correctness, pages 208-238. SpringerVerlag, 1990.

[Hoa84] C.A.R. Hoare. Communicating Sequential Processes. Prentice-Hall, London, 1984.

[Jon83] C.B. Jones. Tentative steps towards a development method for interfering programs. ACM Transactions on Programming Languages and Systems, $5(4): 596-619,1983$.

[KMP93] Y. Kesten, Z. Manna, and A. Pnueli. Temporal verification of simulation and refinement. In J.W. de Bakker, W.-P. de Roever, and G. Rozenberg, editors, LNCS 803: A Decade of Concurrency, Reflections and Perspectives, pages 273-346. Springer-Verlag, 1993.

[Lam83] L. Lamport. What good is temporal logic. In R.E.A. Manson, editor, Information Processing 83: Proc. of the IFIP 9th World Congress, pages 657-668. Elsevier Science Publishers, North Holland, 1983.

[Lam94] L. Lamport. The temporal logic of actions. ACM TOPLAS, 16(3):872-923, May 1994. 
[MC81] J. Misra and K.M. Chandy. Proofs of networks of processes. IEEE Transactions on Software Engineering, 7(4):417-426, July 1981.

[MP89] Z. Manna and A. Pnueli. An exercise in the verification of multi-process programs. Technical report, Stanford University, 1989.

[Sta84] E.W. Stark. Foundations of a Theory of Specification for Distributed Systems. PhD thesis, Massachusetts Inst. of Technology, 1984. Available as Report No. MIT/LCS/TR-342.

[ZCdR92] J. Zwiers, J. Coenen, and W.-P. de Roever. A note on compositional refinement. In C. B. Jones, R. C. Shaw, and T. Denvir, editors, 5th Refinement Workshop, Workshops in Computing, pages 342-366, London, January 1992. BCS-FACS, Springer Verlag.

This article was processed using the $\mathrm{AT}_{\mathrm{E}} \mathrm{X}$ macro package with LLNCS style 